\title{
Die erweiterte Perspektive der Economie des conventions
}

Im folgenden Kapitel wird die das Forschungsprojekt anleitende Theorieperspektive aufgearbeitet. Diese soll es ermöglichen, die in Kap. 2 dargestellte Forschungslücke theoretisch fundieren und beschreiben zu können und sie stellt die Grundlage für die methodologische und methodische Grundlegung der Forschungsperspektive dar.

Die Economie des conventions (EC) stellt dabei die grundlegende Perspektive dar, welche durch weitere Konzepte wie das der Global-Quality-Chains, der Distributed Cognition oder der Workplace Studies ergänzt wird. Die EC stellt im deutschsprachigen Raum ein verhältnismäßig junges Forschungsprogramm dar (Knoll 2012, S. 21). Sie basiert nicht auf einer einheitlichen Theoriearchitektur und wird deswegen oft als netzwerkartiges Forschungsprogramm beschrieben (Diaz-Bone 2011a, S. 16). Obwohl zentrale und integrierende Theorieelemente bestehen wie dasjenige der Konvention, der methodologische Fokus auf Situationen oder die grundlegende Transdisziplinarität (Eymard-Duvernay et al. 2011, S. $3 \mathrm{ff}$.), welche eine grundlegende Basis für dieses Forschungsprogramm darstellen, existiert doch eine Vielzahl an unterschiedlichen Spezifizierungen und Forschungsrichtungen, welche in einer Gesamtschau kein einheitliches Theoriegebäude ergeben (Diaz-Bone 2011a, S. 10). ${ }^{1}$ In der Konsequenz wird es dadurch

\footnotetext{
${ }^{1} \mathrm{Am}$ prominentesten ist wohl die bis heute nicht genügend geklärte Theoretisierung des Verhältnisses der beiden Hauptwerke der EC „Worlds of Production“ (Storper und Salais 1997) und „Über die Rechtfertigung“ (Boltanski und Thévenot 2007) zueinander (Brandl und Pernkopf-Konhäusner 2015, S. 320). Rallet kommentierte die Entwicklung der EC, als interessant und perplex zugleich. Interessant sei sie, weil sie soziologische und ökonomische Perspektiven reintegriere. Perplex mache sie, weil die unter dem Label „EC“ zusammengefasste Forschung doch sehr unterschiedlich sei (1993, S. 45).
} 
notwendig, im folgenden Theoriekapitel nicht nur eine Wahl im Hinblick auf das Forschungsprogramm, sondern darüber hinaus auch innerhalb dieser Perspektive eine Wahl bezüglich der verwendeten theoretischen Werkzeuge vorzunehmen.

Die Ursprünge der EC sind im französischen Statistikamt, dem INSEE, ${ }^{2}$ zu suchen. Hier traf Laurent Thévenot auf Pierre Bourdieu und Luc Boltanski, welche die sozioprofessionellen Kategorien des INSEE zum Forschungsgegenstand gemacht hatten (Dosse 1999, S. 38). Die Forschung an den statistischen Kategorien stand damals noch unter dem Stern der kritischen Soziologie Bourdieus, der auch einen beträchtlichen Einfluss auf die späteren Vertreter der EC hatte (Desrosières 2011, S. 66 ff.; Diaz-Bone 2018, S. 47 ff.). Doch bereits früh kritisierten Boltanski und Thévenot die Soziologie Bourdieus. In ihren Studien zum Kodifizieren im INSEE stellten Boltanski und Thévenot eine höhere Reflexivität der Kodifizierer in der Anwendung der sozioprofessionellen Kategorien fest, als ihnen von der bourdieuschen Theorie zugeschrieben wurde. Vertreter der EC kritisierten die Starrheit des Habitus und wiesen in der Folge das Habituskonzept von Bourdieu zurück (Diaz-Bone 2011a, S. 17). ${ }^{3}$ Kritisiert wurde dabei die Rückführung von Handeln auf lediglich vorreflexive Dispositionen. Dadurch werde die Reflexivität, d. h. die situative Angepasstheit des Handelns durch den Bezug auf verschiedene Konventionen, von Akteuren unterschätzt und nicht gesehen (Thévenot 2011e, S. 43). Die empirisch festzustellende und über verschiedene Klassengrenzen hinaus funktionierende Koordination verweise auf kollektive Logiken der Koordination (Boltanski 2004). Boltanski und Thévenot forderten in der Folge, die situativen Kompetenzen und Interpretationsleistungen der Akteure ernst zu nehmen, was in einer stärkeren pragmatischen Theoriefundierung endete (Boltanski 2010, S. 74; Boltanski und Thévenot 2011, S. 50; Thévenot 2011a, S. 386). ${ }^{4}$ Die EC wurde dabei oft als pragmatische Soziologie (Dodier 2011; Nachi 2006), bzw. als pragmatisch-strukturalistische Soziologie (Diaz-Bone 2011b) bezeichnet. Der tatsächliche Bezug auf Arbeiten der nord-

${ }^{2}$ Für einen breiten Überblick über die Gründungsmomente der EC vgl. Diaz-Bone (2018, S. 13 ff.).

${ }^{3}$ Wobei eben auch Gemeinsamkeiten und gegenseitige Steigerungsmöglichkeiten zwischen der bourdieuschen Soziologie und der EC bestehen (Benatouil 1999; Diaz-Bone 2007; Vogel 2015).

${ }^{4}$ Zudem sah beispielsweise Boltanski das Potential der bourdieuschen Soziologie als ausgeschöpft an: „When Distinction appeared, I was in the middle of writing Les Cadres. I told myself that there was nothing left in that paradigm“ Boltanski zitiert nach Dosse (1999, S. 37). 
amerikanischen Pragmatiker wie Peirce, James und Dewey fand jedoch gemäß Joan Stavo-Debauge erst relativ spät statt (2013). Mit dem Rückgriff auf pragmatische Forschungswerkzeuge ist die EC im deutschsprachigen Raum nicht allein. Ein weiteres Beispiel stellen die neo-pragmatischen Arbeiten von Hans Joas dar (1992, 2002). Zudem schlug Jens Beckert vor, die handlungstheoretische Grundlegung der Wirtschaftssoziologie im amerikanischen Pragmatismus und seinen Weiterentwicklungen zu suchen (2009, S. 7). Die Absetzung von Bourdieu ging parallel zur Auseinandersetzung mit der sich etablierenden Actor-Network-Theory (kurz: ANT). ${ }^{5}$ Diese ist auf der Basis von frühen Laborstudien von Latour entwickelt worden (Latour 1987) und hat sich zu einer Theorie von sozio-technischen Netzwerken entwickelt (Latour 2007, 2008; Callon 2006). Eine gemeinsame Grundlage stellte dabei die intensive Auseinandersetzung mit der Koordinationsfunktion von Objekten dar (Diaz-Bone 2011a, S. 12, 2018).

Die frühen Arbeiten von Boltanski, Desrosières und Thévenot zur Statistik ${ }^{6}$ und zum Kodieren spielten eine wichtige Rolle für die entstehende EC (Corcuff 2011; Desrosières 2008a, b, 2014; Diaz-Bone 2018; Diaz-Bone und Didier 2016a; Dosse 1999). Zudem wurde mit der Einführung des Äquivalenzprinzips als ein die Kategorisierung organisierendes Prinzip durch Alain Desrosières auch ein frühes Konventionenkonzept vorgelegt (Diaz-Bone 2011a, S. 20). Durch die Arbeiten zur Logik der Klassifikation und der Codierung entwickelte sich die Erforschung von Kategorien(-ordnungen) und deren Genese zu einem zentralen Forschungsfokus der EC (Latsis 2006, S. 258).

In der nun folgenden Darstellung der Forschungsperspektive wird zuerst ein Überblick über zentrale Konzepte der EC geliefert. Danach werden Produktionswelten als übergreifendes Konzept eingeführt, welches es ermöglicht, die bisher dargestellten Konzepte zu einer kohärenten Forschungsperspektive zu verdichten. Darauf folgend wird auf die Wertigkeitsproduktion von Produkten aus einer EC-Perspektive eingegangen. Auf der Basis der verschiedenen theoretischen Konzepte wird im abschließenden Unterkapitel die Forschungsperspektive formuliert und zu forschungsleitenden Fragen verdichtet. Das Ziel liegt darin, die in der Einleitung aufgeworfenen Forschungsfragen mithilfe der Theorieperspektive zu präzisieren.

\footnotetext{
${ }^{5}$ In der Folge wurden die beiden Theorierichtungen als die neuen französischen Sozialwissenschaften bezeichnet (Diaz-Bone 2011a, S. 9 f.).

${ }^{6}$ Unter Statistik versteht Desrosières folgendes: „By ,statistics“ we mean here the body of knowledge consisting of the registration and presentation of quantitative data and the algorithms for its analysis such as series, indicators, econometric models, and many other methods available nowadays in databanks and data-processing packages“" (2009b, S. 43).
} 


\subsection{Situation und Koordination}

In den folgenden Unterkapiteln werden die für die EC zentralen Konzepte der Situation und der Koordination vorgestellt. Zentral für das Verständnis beider eng miteinander verwandten Konzepte ist die Dezentrierung des handelnden Subjekts in der EC. Der Ursprung von „Handlung“ wird folglich nicht mehr alleine in den Intentionen von Subjekten gesucht, „Handlung“ wird vielmehr als Ergebnis eines Prozesses der Auseinandersetzung mit einer materialen und sozialen Umwelt verstanden (Eymard-Duvernay et al. 2011). Laurent Thévenot schlägt aus diesem Grund den Begriff der Koordination vor, da damit der Prozess der Auseinandersetzung mit der materiellen und sozialen Umwelt betont wird (Thévenot 2011c). Die Dekonstruktion des handelnden Subjekts als Analyseeinheit führt zu einem methodologischen Fokus auf Situationen als grundlegender Analyseeinheit (Diaz-Bone 2018). Denn die Rückweisung von Subjekten als alleiniger Handlungsinstanz macht einen erweiterten Fokus der Analyse auf weitere Situationselemente (Objekte, Formen und Konventionen) notwendig.

\subsubsection{Regime des Engagements}

Ein für die EC zentrales Werk stellt „Über die Rechtfertigung“ von Luc Boltanski und Laurent Thévenot dar (2007). In diesem Werk stellen die beiden Autoren sechs sog. Rechtfertigungsordnungen vor, welche als kollektive Handlungsgrammatiken verstanden werden können (vgl. hierfür ausführlich Abschn. 3.1.1). Diese dienen als Basis für die (öffentliche) Handlungskoordination und Rechtfertigung. Bereits kurz nach dem Erscheinen von „Über die Rechtfertigung“", arbeitete Thévenot an der Konzeptualisierung von Handlungslogiken, welche nicht auf die durch Boltanski und ihn identifizierten Rechtfertigungsordnungen Bezug nehmen und somit nicht einem Rechtfertigungsdruck ausgesetzt sind. ${ }^{8}$ Theoriehistorisch stellt das

\footnotetext{
${ }^{7}$ Unter dem französischen Titel „De la justification“ (Boltanski und Thévenot 1991).

${ }^{8}$ Bereits im Nachwort zu „Über die Rechtfertigung“ sprechen Boltanski und Thévenot von der Notwendigkeit eines Analyserahmens, welcher nicht nur die Rechtfertigung miteinschließt (Boltanski und Thévenot 2007, S. 464). Ebenfalls eine Theorie der Handlungsregime hat Luc Boltanski eingeführt. Allerdings ist diese nicht als vertikale Differenzierung der Rechtfertigungstheorie ausgelegt, sondern bezieht sich auf Handlungsregime wie diejenigen der Liebe und der Gewalt, die nicht Äquivalenzbemühungen unterliegen (Boltanski 2012, S. 68 ff.).
} 
Konzept der Regime des Engagements eine Fortführung von „Über die Rechtfertigung“ dar. Die Unterscheidung von verschiedenen Regimen des Engagements stellt jedoch einen zentralen Beitrag zur Handlungstheorie der EC dar und wird aus diesem Grund hier entgegen der Theoriehistorie eingeführt. ${ }^{9}$ Thévenot unterscheidet in der Regimetheorie zwischen verschiedenen sog. „Regimen des Engagements“. 10 Thévenot führte neben dem Rechtfertigungsregime das Regime des planenden Handelns und das Regime des Vertrauten ein (2011c, d). ${ }^{11}$ Nicolas Auray führte anschließend an Thévenot das Regime der Exploration ein (Auray 2011). Jede Person ist zu verschiedenen Zeitpunkten in den Regimen engagiert. ${ }^{12}$ Handlungsregime sind durch eine eigene Realität gekennzeichnet. Diese spezifische Realität ist gekoppelt an ein spezifisches „Gut“, d. h. an ein spezifisches Versprechen, welches im jeweiligen Handlungsregime erreicht werden will (Thévenot 2011c, S. 232 f.). ${ }^{13}$ Das jeweilige Gut ist für Thévenot das Prinzip, das in einer Situation verwirklicht werden soll. Zur Erreichung der spezifischen Güter der

${ }^{9}$ Zugleich wurden von Thévenot bereits zu Beginn der 90er Jahre verschiedene Handlungsregime differenziert (Thévenot 1990).

${ }^{10}$ Die Theorie der Handlungsregime wird bis jetzt nur zögerlich für empirische Arbeiten im deutschsprachigen Raum verwendet, trotz der Anerkennung von deren Relevanz einerseits für die Erweiterung der Handlungstheorie der EC (Knoll 2013, S. 374 ff.) und andererseits generell für die soziologische Konzeptualisierung von „Handlung“ (Nicolae 2014).

${ }^{11}$ Thévenot versteht die Regimetheorie als integrative Handlungstheorie. Gemäß ihm korrespondieren die verschiedenen Handlungsregime mit verschiedenen sozialwissenschaftlichen Handlungstheorien. Unterschiedliche Theorieansätze präferierten demnach verschiedene kognitive Handlungsformate. Er sieht im Rechtfertigungsregime kollektive Handlungslogiken am Werk, welche von Durkheim in die Soziologie eingeführt wurden. Das Regime des Plans sieht er vor allem durch Rational Choice Theorien theoretisch ausgearbeitet. Das Regime des Vertrauten sieht er in phänomenologischen Theorieansätzen beschrieben (Thévenot 2006a, S. 211).

${ }^{12}$ Thévenot spricht deswegen von einer „Schubladenpersönlichkeit“ (franz.: „Une personalité à tiroir") (2006a, S. 261 ff.), da sich Personen nicht durch ein Sozialisierungsprinzip, sondern durch Sozialisationen in mehreren Regimen unterscheiden (Thévenot 2006a, S. 233 ff.).

${ }^{13}$ Obwohl das Konzept der Handlungsregime von Thévenot bereits relativ früh entworfen wurde (Thévenot 1990), wird es doch bis jetzt kaum als Schritt zu einer umfassenden Handlungstheorie der EC wahrgenommen und rezipiert. Dies liegt wohl nicht zuletzt am Fehlen einer „,echten“ Monographie - „L'action au pluriel“ stellt eine Zusammensetzung von verschiedenen Aufsätzen dar (Thévenot 2006a) - und an der Zerstreutheit der Theorie über viele Aufsätze hinweg. 
verschiedenen Regime sind unterschiedliche Arten des Engagements notwendig. Thévenot benutzt bewusst den Begriff des Engagements anstelle des Handelns. Denn das Engagement drückt das „In der Welt sein“ von Akteuren aus (Centemeri 2015, S. 305), das heißt die Auseinandersetzung mit der durch Objekte strukturierten Umwelt und die in den verschiedenen Regimen unterschiedliche Agency (Thévenot 2011d, S. 256). ${ }^{14}$ Zentral ist für Thévenot in allen Regimen die Abstützung durch Objekte. Insbesondere im Regime des Vertrauten sieht er die Agency als sehr eng zwischen Objekten und Subjekten verflochten an (Thévenot 2011c, S. 238). Zudem ergibt sich durch die Konzeption von verschiedenen Regimen des Handelns ein diskontinuierlicher Unterschied zwischen dem Privaten und dem Öffentlichen (Thévenot 2011a, S. 391).

Die Theorie der Handlungsregime ist für die vorliegende Arbeit aus drei Gründen ein wichtiges theoretisches Konzept. Zunächst sind die unterschiedlichen in den verschiedenen Survey-Welten existierenden Survey-Qualitäten eng mit dem Potenzial von Daten für die Handlung in unterschiedlichen Regimen des Engagements verknüpft. Die Theoretisierung von unterschiedlichen Survey-Qualitäten in dieser Arbeit hängt folglich eng mit dem Konzept der Handlungsregime zusammen. Zusätzlich basieren jedoch auch die Koordinationsprozesse in den verschiedenen Survey-Welten auf unterschiedlichen Regimen des Engagements. Wie zu zeigen sein wird, ist die Unterscheidung von unterschiedlichen Orientierungen der Handlung aber auch hilfreich beim Verständnis von Artikulationshürden bei der Beantwortung von Survey-Fragen.

Thévenot kritisiert auf der Basis der Regime des Engagements das klassische Informationsverständnis. Denn damit etwas als Information wahrgenommen werden kann, bedarf es zuerst entsprechender bereits bekannter Formen, auf welche sich eine Mitteilung beziehen kann, um folgend auch als Information wahrgenommen zu werden. Informationen sind so gemäß Thévenot breit verstehbare und legitime Formen des Wissens (Thévenot 1997). Um von einer breiten Masse verstanden zu werden, müssen persönliche Erlebnisse auf weitreichende Formen Bezug nehmen (Thévenot 2006a, S. 134). ${ }^{15}$ Bei dieser Übersetzung gehen jedoch Eigenheiten der spezifischen persönlichen Erfahrung verloren, da diese quasi normiert werden müssen. Regime können auch im Konflikt zueinanderstehen.

\footnotetext{
${ }^{14}$ Thévenot hat ebenfalls andere Begriffe anstelle der Regime des Engagements gebraucht: „Mode de coordination“, „Regime d'ajustement“" und „Action qui convient“. Die Festlegung auf den Begriff des Engagements wählte Thévenot, um die zentrale Rolle der Objekte in der Stabilisierung von Regimen hervorzuheben (Thévenot 2006a, S. 13).

${ }^{15}$ Dies wird gemäß Thévenot vermehrt von sozialen Bewegungen verwendet, welche folglich unformulierte Anliegen des Regimes des Vertrauten politisch artikulieren (2006a, S. 220).
} 
So zeichnet sich die staatsbürgerliche Rechtfertigungsordnung gerade durch einen Verzicht auf Partikularinteressen aus. Der Bezug auf das Regime des Vertrauten in dieser Rechtfertigungsordnung würde abgestraft (Thévenot 2006a, S. 221). ${ }^{16}$

Thévenot sieht in der Kritik des klassischen, kognitionswissenschaftlichen Informationsbegriffs den Ausgangspunkt der EC (Thévenot 2011d, S. 260). Denn aus Sicht der EC benötigt jede Information auch eine In-Formation, das heißt jede Information muss ins Verhältnis einer Formationslogik gestellt werden, um als solche erkannt werden zu können.

Das implizite Versprechen des Rechtfertigungsregimes ist es, eine allgemein gültige Ordnung zu erreichen. Die Kommunikation ist dadurch eine weit verstandene politische, welche sich auf Objekte von großer Bedeutungsreichweite stützten kann. Koordination in diesem Regime hat dadurch eine sehr große Reichweite (Thévenot 2006a, S. 129). Dieses Regime wird folglich immer dann angestrengt, wenn es um (öffentliche) Fragen der Wertigkeit und Legitimation geht (Thévenot 2011d). Konflikte und Dispute sind in diesem Regime folglich hoch ,politisch“, d. h. stark durch Fragen einer kollektiven Moral gekennzeichnet. Es ist dieses Handlungsregime, welches in der für die EC zentralen Monografie „Über die Rechtfertigung“ ausführlich thematisiert wird (Boltanski und Thévenot 2007). Die verschiedenen Rechtfertigungsordnungen dieses Handlungsregimes werden in Abschn. 3.1.1 ausführlicher dargestellt. ${ }^{17}$

Das implizite Versprechen des Regimes des planenden Handelns liegt im Erreichen eines Ziels. Das Regime des planenden Handelns ist gemäß Thévenot so alltäglich, dass es als analytisches Konzept für die Soziologie droht, vergessen

\footnotetext{
${ }^{16}$ Thévenot entwickelte im Nachgang zu seiner Theorie der Handlungsregime eine darauf basierende Theorie der politischen-kulturellen Systeme. War die Theorie der Rechtfertigungsregime zunächst als generelle Theorie des Politischen gedacht, argumentierte Thévenot später, dass sich unterschiedliche politisch-kulturelle Systeme anhand der vorherrschenden Regime-Kommunikation einteilen lassen. Frankreich steht bei ihm dann als Modell für die politische Kommunikation im Rechtfertigungsregime, die USA für das Regime des planenden Handelns und Russland für das Regime des Handelns im Vertrauten. Politische Kommunikation in den USA basieren folglich weniger auf der Idee einer Definition einer gemeinsamen Wertgrundlage, denn auf der politischen Kommunikation als individuelle Willensäußerung im Sinne eines „Public Choice“. Diese Eigenarten von Systemen zeigen sich dann beispielsweise auch in pädagogischen Einrichtungen: Gilt es in Frankreich gemäß Thévenot beim Aufsatzschreiben, eine Lektüre im Hinblick auf generelle Begriffe und Argumente zu untersuchen, so ist es beispielsweise in Russland eher das Ziel, den persönlichen Eindruck der Lektüre wiederzugeben (Thévenot 2014b, S. 20 f.).

${ }^{17}$ Eine Systematisierung der Relationen zwischen Regimen des Engagements einerseits und zwischen Regimen des Engagements und Rechtfertigungsordnungen andererseits wird im Postscriptum dieser Arbeit vorgenommen.
} 
zu werden (Thévenot 2011c, S. 240). Es ist charakterisiert durch ein funktionales Verhältnis zur Umgebung, welche durch autonome Akteure auf Ressourcen für die Erfüllung eines Ziels (eines Interesses) befragt wird. Die Kommunikation orientiert sich hier funktional an der Erreichung dieses vorgenommenen Plans und basiert auf verbaler Sprache (Thévenot 2006a, S. 119). Deswegen findet sich dieses Regime auch im Herzen einer industriellen Organisation (Thévenot 2006a, S. 114) ${ }^{18}$ Die Einführung des Regimes des planenden Handelns kann als Korrektur einer ,übermoralisierten“ Handlungskonzeption im Nachgang von „Über die Rechtfertigung“ gelesen werden. Denn dem Handlungsmodell der Rechtfertigungstheorie folgend, ist Handlung generell auf weitreichend legitimierte Rechtfertigungsordnungen aufgebaut. Mit Bezug auf das Regime des planenden Handelns lässt sich dagegen auf die Besonderheit des rechtfertigenden Handelns im alltäglichen Handlungsstrom verweisen.

Das implizite Versprechen des Regimes des Vertrauten liegt in der Annehmlichkeit, bzw. im Komfort des Bekannten und Vertrauten. Das Regime des Vertrauten zeichnet sich durch ein Suchen nach Annehmlichkeit aus, bzw. durch den Eindruck der höchst subjektiven eigenen Erfahrung. Die Handlung in diesem Regime ist stark durch Objekte mitstrukturiert durch die enge, vertraute Beziehung zwischen Akteur und Objekten. ${ }^{19}$ Agency kann deshalb kaum mehr klar einzelnen Akteuren oder Objekten zugeschrieben werden (Thévenot 2006a, S. 120). ${ }^{20}$ Marc Breviglieri spricht deshalb von der „Handhabung“ als der Agency-Form dieses Regimes (Breviglieri 2004). ${ }^{21}$ Die Kommunikation im

\footnotetext{
${ }^{18}$ Auch wenn organisationale Dispositive maßgeblich auf Dispositiven der Rechtfertigungsordnungen basieren (Thévenot 2006a, S. 136).

${ }^{19}$ In der Regimetheorie bis jetzt nicht thematisiert ist das Verhältnis zwischen Tier und Mensch. Colin Jerolmack zeigt mit seiner Ethnographie von türkischen Taubenhaltern in Berlin jedoch eindrücklich die Stützung des Vertrauten durch Tiere, bzw. Tierhaltung auf (Jerolmack 2007). Eine regimetheoretisch spannende Kategorisierung stellt dabei die Differenz zwischen Haus- und Nutztieren dar.

${ }^{20}$ Die starke Stützung des Vertrauten durch Objekte wird beispielsweise von Daniel Miller untersucht (2010).

${ }^{21}$ Breviglieri schlägt den Begriff der „Handhabung“ anstelle des „Brauchs“ vor, um das Aktive im Regime des Familiären zu unterstreichen (Breviglieri 2004, S. 24). Wenn im weiteren Text von der Handhabung die Rede ist, soll zunächst diese aktive Bewohnbarmachung der Umgebung, d. h. das Erstellen einer belebbaren, vertrauten Umgebung beschrieben werden. Thévenot folgend kann aber argumentiert werden, dass in der Folge von geöffneten und geschlossenen Augen in jedem Regime, das Regime des Familiären auch eine blinde Seite hat (Thévenot 2013, S. 172). Diese blinde Seite wird hier als Widerständigkeit/Unangepasstheit gegenüber anderen Regimen konzipiert.
} 
Regime des Vertrauten ist größtenteils nonverbal ${ }^{22}$ und geht weit über das diskursive Sprechen hinaus. Denn zusätzlich zum Sprechen kommt hier der Körper als Kommunikationsmedium zum Einsatz (Thévenot 2011c, S. 240). Thévenot weist darauf hin, dass das gesprochene Wort die Intimität des Vertrauten bereits beträchtlich abwandelt (Thévenot 2011d, S. 267). Denn das hier gewonnene Wissen wird durch einen Trial-and-Error-Prozess gewonnen (Thévenot 2011d, S. 266). Der Grund für die Schwierigkeit der verbalen Kommunikation liegt in den von Person zu Person unterschiedlichen Sozialisierungserfahrungen (Thévenot 2007, S. 416). ${ }^{23}$

Die spezifische Kommunikationsart des Regimes des Vertrauten lässt sich auch durch den Bezug auf das ethnomethodologische Konzept des „Groundings“ aufschlüsseln. Das Konzept des Groundings verweist auf den Umstand, dass Sinn immer situativ hergestellt, das heißt ein gemeinsamer Grund erarbeitet werden muss (Clark und Brennan 1991). Dies kann abhängig von der Situation in unterschiedlichem Masse notwendig sein. Bezieht man das Konzept des Groundings auf die unterschiedlichen Kommunikationsarten in Regimen, so werden die unterschiedlichen Voraussetzungen der Kommunikation deutlich. Denn es ist gerade das intime Verständnis der eigenen Persönlichkeit durch jemand Vertrauten, welche das Versprechen der Annehmlichkeit im Regime des Vertrauten auch in Anwesenheit weiterer Personen ermöglicht. Indexikalität als kommunikative Herausforderung (Garfinkel 1984, S. 4 ff.) wird in diesem Regime folglich durch die gegenseitige Vertrautheit von Erfahrungen und das gegenseitige Verstehen ${ }^{24}$ und sich davon speisenden Begriffen zumindest teilweise umgangen. Es ist dieser Umstand, der die fast sprachlose Kommunikation in diesem Regime ermöglicht.

\footnotetext{
${ }^{22}$ Thévenot bezog das Regime des Vertrauten mehrfach auf die bourdieuschen Konzepte der Praktiken und des Habitus. Aus der Sicht Thévenots stellen die bourdieuschen Konzepte jedoch lediglich die blinden Seiten des Regimes des Vertrauten dar, da sie auf unbewusste Praktiken fokussierten. Dagegen sieht Thévenot im Regime des Vertrauten eben auch ein aktives Engagement zum Erreichen von Annehmlichkeit. Zusätzlich gehe Bourdieu von einer übertriebenen Annahme der Kollektivität von Praktiken aus (Thévenot 2009b, S. 803, 2011b, S. 196 f.).

${ }^{23}$ Hier lässt sich aber auch festhalten, dass auch innerhalb des Vertrauten eine Kollektivität der Erfahrung festgehalten werden kann. Dies zeigt sich besonders deutlich in der Studie von Colin Jerolmack über türkischstämmige Taubenhalter in Berlin (2007). Hier wird die durch die Migration sichtbare kollektive Stützung des Vertrauten durch die Tierhaltung sichtbar, welche eine Differenz zu den restlichen Berlinern darstellt.
}

${ }^{24}$ Im Sinne Mannheims (1980, S. 272 f.). 
Mit Karl Mannheim lässt sich argumentieren, dass in diesem Regime eine konjunktive Erfahrung 25 stattfindet (Bohnsack 2014, S. 61 ff.; Mannheim 1980, S. 211 ff.). Mannheim bezeichnet das Nachvollziehen von konjunktiver Erfahrung als „Verstehen“, während er das Nachvollziehen von generellen Begriffen als „Interpretieren“ bezeichnet (Mannheim 1980, S. 272 f.). Das Regime des Vertrauten ist dementsprechend der Ort der Einzelmeinungen, der Subjektivitäten, also das Regime mit dem geringsten Anteil an gemeinsamen kognitiven Rahmen (Basto und Centemeri 2014, S. 171). Dies ist durch einen für jede Person individuellen und lokalen Habitualisierungsweg begründet (Thévenot 2015, S. 209).

Das Regime der Exploration wurde von Nicolas Auray eingeführt (2007). Das hier investierte Gut stellt die Spannung für Neues dar. Das Informationsformat dieses Regimes ist die Überraschung, d. h. Handlung wird hier danach bewertet, inwiefern sie Überraschungen ermöglicht. Es ist dadurch nicht überraschend, dass Auray dieses Regime anhand moderner Kommunikations- und Informationstechniken wie Google, Wikipedia, usw. entworfen hat (Auray 2011). Denn diese ermöglichen es mit wenig Aufwand, neue Welten zu entdecken. Die Voraussetzung hierfür ist es jedoch, die Gedanken schweifen zu lassen und nicht auf geplante Handlungen fokussiert zu sein (Auray 2011, S. 331). Kompetenzen in diesem Regime stellen Kreativität und das Verfügen über einen Entdeckergeist dar (Thévenot 2014a, S. 134). Auch wissenschaftliches Arbeiten weist Bezüge zu diesem Regime auf. Denn die Erkenntnis von Neuem lässt sich nicht im Vornhinein planen. ${ }^{26}$

\footnotetext{
${ }^{25}$ Mannheim schreibt über die Eigenart der konjunktiven Erfahrung: „Was den Geltungsbereich konjunktiver Erkenntnis betrifft, so gilt diese zunächst nur für mich, wenn ich ein Ding erkenne. Ist mein Erkenntnisobjekt ein Mensch, so ist - wie dies im zuerst analysierten Beispiel sichtbar wurde - das perspektivische Bild (die mir zugewandte Seite seines seelischen Selbstes) auf Grund der existentiellen Beziehung, in der wir stehen, aus meinem Wissen in das seinige übertragbar, wie auch umgekehrt er mir sein Wissen von mir mitteilen kann. (Dies geschieht sehr häufig auch ohne Worte, aber auch wenn Worte verwendet werden, erschöpfen diese niemals den übertragenen Gehalt, der uns gerade kraft der lebendigen Beziehung, in der wir gemeinsam existieren, den großen Teil der Formulierungen erspart.) Aber selbst wenn dieses Wissen vom anderen noch so reich ist, erkennt keiner von uns beiden den anderen so, wie er hypothetischerweise,,an sich"sein mag. Jeder erkennt vom anderen nur so viel, und nur in der Weise, wie er in unsere gemeinsame Beziehung eingeht, in ihr existiert, in ihr sich entfaltet.“ (1980, S. 213) und: „Das Erfahrene ist durch und durch persönlich, auch wenn es wie in unserem Falle zweipersonal verankert ist; es ist „Dritten“"nicht ohne weiteres mitteilbar, es sei denn, dass diese durch eine Einweihung in unsere Beziehung, durch Aufnahme von Existenz in Existenz sich mit uns verbinden“ (ebd., S. 215).

${ }^{26}$ Ein Konzept, welches das Engagement in diesem Regime in der Wissenschaft, genauer der qualitativen Sozialforschung, beschreibt, stellt die Methode der Abduktion dar (Reichertz 2013).
} 
Die verschiedenen Regime des Engagements können anhand mehrerer Unterscheidungsdimensionen miteinander verglichen werden. In Tab. 3.1 wird ein Überblick über die Unterschiede zwischen den verschiedenen Regimes des Engagements dargestellt.

Tab. 3.1 Die verschiedenen Regime des Engagements

\begin{tabular}{|c|c|c|c|c|}
\hline & $\begin{array}{l}\text { Rechtfertigungs- } \\
\text { regime }\end{array}$ & $\begin{array}{l}\text { Regime des pla- } \\
\text { nenden Handelns }\end{array}$ & $\begin{array}{l}\text { Regime des } \\
\text { Vertrauten }\end{array}$ & $\begin{array}{l}\text { Regime der } \\
\text { Exploration }\end{array}$ \\
\hline Investiertes Gut & $\begin{array}{l}\text { Beitrag zum } \\
\text { Gemeinwohl }\end{array}$ & $\begin{array}{l}\text { Erreichter eigener } \\
\text { Wille }\end{array}$ & $\begin{array}{l}\text { Persönliche } \\
\text { Annehmlich- } \\
\text { keit, Komfort }\end{array}$ & $\begin{array}{l}\text { Spannung für } \\
\text { Neues }\end{array}$ \\
\hline $\begin{array}{l}\text { Informations- } \\
\text { format }\end{array}$ & $\begin{array}{l}\text { Rechtfertigungs- } \\
\text { basiert }\end{array}$ & Funktional & Teilnahmsvoll & Überraschung \\
\hline $\begin{array}{l}\text { Form des Tests/ } \\
\text { Realitätszugang }\end{array}$ & $\begin{array}{l}\text { Feststellen } \\
\text { der Größe im } \\
\text { Hinblick auf } \\
\text { Rechtfertigungs- } \\
\text { ordnungen }\end{array}$ & $\begin{array}{l}\text { Feststellen } \\
\text { des normalen } \\
\text { Funktionierens } \\
\text { von Handlungen/ } \\
\text { Prozessen }\end{array}$ & $\begin{array}{l}\text { Lokal und } \\
\text { persönlich }\end{array}$ & $(-)$ \\
\hline Zeitlichkeit & $\begin{array}{l}\text { Unterschiedlich, } \\
\text { abhängig von der } \\
\text { jeweiligen Recht- } \\
\text { fertigungsordnung }\end{array}$ & $\begin{array}{l}\text { Zukunfts- } \\
\text { projektion }\end{array}$ & $\begin{array}{l}\text { Vergangenheits- } \\
\text { orientierung }\end{array}$ & $(-)$ \\
\hline $\begin{array}{l}\text { Gegenseitiges } \\
\text { Engagement }\end{array}$ & $\begin{array}{l}\text { Kollektiv für das } \\
\text { gemeinsame Gut }\end{array}$ & $\begin{array}{l}\text { Gemeinsames } \\
\text { Projekt, durch } \\
\text { Absprachen, } \\
\text { Verträge }\end{array}$ & $\begin{array}{l}\text { Einfühlsam } \\
\text { (liebend, } \\
\text { freundlich, } \\
\text { intim), in } \\
\text { Freundschaft }\end{array}$ & Spielend \\
\hline $\begin{array}{l}\text { Anderen helfen } \\
\text { sich zu enga- } \\
\text { gieren }\end{array}$ & $\begin{array}{l}\text { Auf öffentlichen } \\
\text { Test Vorbereiten }\end{array}$ & $\begin{array}{l}\text { Den Willen von } \\
\text { jemandem stärken, } \\
\text { das Vertrauen in } \\
\text { jemandes Plan } \\
\text { stärken }\end{array}$ & $\begin{array}{l}\text { Vertrauen geben } \\
\text { durch persön- } \\
\text { liche Aufmerk- } \\
\text { samkeit }\end{array}$ & $(-)$ \\
\hline Kompetenz & $\begin{array}{l}\text { Rhetorik; Arrange- } \\
\text { ment-Fähigkeiten }\end{array}$ & $\begin{array}{l}\text { „Handeln“; } \\
\text { Umsetzen; } \\
\text { Macherqualitäten } \\
\text { besitzen }\end{array}$ & $\begin{array}{l}\text { Empathie; } \\
\text { persönliche } \\
\text { Kenntnis }\end{array}$ & $\begin{array}{l}\text { Kreativität; } \\
\text { Entdecker- } \\
\text { geist }\end{array}$ \\
\hline
\end{tabular}

Quelle: (Auray 2007; Auray und Vétel 2013; Thévenot 2013, 2014a) 
Thévenot konzipiert ein Stufenmodell der Regime: Für ein souveränes Handeln in „höheren“ Regimen ist eine stabile Erfahrung der ,niedrigeren“ Regime notwendig (Thévenot 2011c, S. 237). Zudem sind Situationen nicht zwingend nur durch den Modus eines Regimes charakterisiert. Nebst dem, dass Akteure stets den Koordinationsmodus festlegen müssen, können Situationen auch auf unterschiedlichen Regimen basieren im Sinne einer Bricolage ${ }^{27}$ (Thévenot 2013, S. 163). Thévenot nennt als Beispiel das Gericht, welches nebst der rechtfertigungsbasierten Rechtsprechung auch auf den Modus des autonomen Handelnden als Orientierungsfigur Bezug nimmt (Thévenot 2006a, S. 180).

Die verschiedenen Handlungsregime unterscheiden sich im Hinblick auf ihre Bewertung von Mitmenschen und Objekten. Sie unterscheiden sich jedoch auch im Hinblick auf ihre Lernprozesse und Sozialisierungen (Thévenot 2011c, S. 253). Die Frage nach der Sozialisation eines Menschen stellt sich damit nicht in einer Gesamtschau für eine Person, sondern muss Thévenot folgend für jedes Regime einzeln gestellt werden (Thévenot 2006a, S. 237). Damit folgt, dass Lernprozesse in einem Regime nicht automatisch Anpassungsprozesse in einem anderen Regime mit sich ziehen. Akteure sind dadurch potentiell intern stark differenziert.

Die Ausführungen Thévenots zur Regimetheorie zielen maßgeblich auf die Schwierigkeiten der Übersetzung zwischen Regimen. Analytisches Potential hat die Theorie der Handlungsregime entsprechend maßgeblich im Darstellen der Transzendierung von Logiken über Regime hinweg mit entsprechenden Brüchen in der Transformation (Thévenot 2006a, S. 129). Als Beispiel verweist er auf die Herausforderung der Sozialen Arbeit, Rechtfertigungskonventionen nicht nur in effektive Handlungsprogramme zu übersetzen, sondern diese auch in der vertrauten Umgebung der Klienten wirksam werden zu lassen, ohne dabei den ursprünglichen politischen Auftrag zu vergessen (Thévenot 2011c, S. 245). Zwei Hürden sind also zu überwinden: Einerseits müssen Regeln gefunden werden (bzw. Formen bei Thévenot), um eine Handlungsorientierung für Sozialarbeitende zu schaffen, das heißt greifbar zu machen. Andererseits müssen diese Formen auch an die Klienten herangetragen werden, wobei auf das Gut des Vertrauten der Person eingegangen werden muss. Hier ist es insbesondere das soziale

\footnotetext{
${ }^{27}$ Hier kann kritisch angefragt werden, ob Regime untereinander auch eine Hierarchie bilden. Die Idee Thévenots, wonach ,höhere“ Regime auf einer soliden Erfahrung im Regime des Vertrauten aufbauen (2011c, S. 237), kann als Hinweis für eine solche Auslegung gedeutet werden. Klar ist jedoch, dass Thévenot infolge der Absetzung von Bourdieu nicht an eine absolute Dominanz eines Regimes denkt. Dies zeigt sich insbesondere in der Autonomie von Lernprozessen in den verschiedenen Regimen (Thévenot 2006a, S. 237).
} 
Fingerspitzengefühl der Sozialarbeitenden, das dazu beiträgt, dass die Soziale Arbeit nicht als „kalte Technokratie“ wahrgenommen wird. ${ }^{28}$ Aus der Perspektive der Regime des Engagements wird so insbesondere der Prozess der Beantwortung von Survey-Fragen problematisiert. Denn hierbei werden nicht lediglich Informationen ,abgerufen“, sondern der Prozess der Artikulation von Informationen stellt vielmehr eine komplexe Koordination zwischen verschiedenen Regimen des Engagements dar. So müssen insbesondere die Eigenheiten und Subjektivitäten des Regimes des Vertrauten in das Regime der Rechtfertigung übersetzt werden. Dies wird erforderlich durch die Notwendigkeit des Bezugs der individuellen Verhältnisse auf die allgemeinen Kategorien der Befragung. Es muss zudem eine Koordination mit dem Regime des Plans stattfinden im Hinblick auf das praktische Ausfüllen des Fragebogens. Zudem wird die Koordination mit dem Regime der Exploration zunehmend zu einem Thema in der Survey-Forschung. Durch die sinkende Teilnahmebereitschaft an Surveys werden neue Wege gesucht, Personen zur Teilnahme an Befragungen zu bewegen. Eine Strategie stellt hierbei die sog. „Gamification“ dar, d. h. der spielerische Gestaltung der Beantwortung von Fragebögen (Unger 2015).

\subsubsection{Konventionen}

Wie in der Einleitung dieses Kapitels deutlich gemacht wurde, ist die EC durch eine gewisse interne Heterogenität gekennzeichnet. Sie ist deswegen eher eine Wissenschaftsbewegung, denn ein Forschungsparadigma. ${ }^{29}$ Das zentrale integrierende Konzept ist dabei das der Konvention (Diaz-Bone 2018, S. 3 f.). Die Konzeptualisierung des Konventionenkonzepts weist dabei einige Unterschiede zur alltagssprachlichen Verwendung des Konventionenbegriffs auf. So sind Konventionen aus der Perspektive der EC nicht einfach Bräuche oder Regeln für das Handeln. Sie stellen vielmehr kollektiv geteilte Handlungslogiken dar, welche für die Überwindung von Unsicherheiten referenziert werden. Zudem unterscheidet

\footnotetext{
${ }^{28}$ Vgl. hierzu auch die Studie von Eduardo Basto und Laura Centemeri zur Schwierigkeit der Übersetzung von globalen Politiken der Küstenerosion in das Handeln im Vertrauen von Küstenbewohnern (2014).

${ }^{29}$ Diaz-Bone verweist auf die dezentrierte, d. h. auf viele Forscher verteilte Theorieentwicklung der EC. Forschung wird so nicht mehr um eine zentrale Forscherpersönlichkeit herum organisiert wie etwa in der bourdieuschen Soziologie, sondern durch ein Netzwerk an Forschern, was im Endeffekt eine höhere Heterogenität zur Folge hat (2011a).
} 
sich das Konventionenkonzept der EC deutlich vom alltagssprachlichen Gebrauch von Konventionen im Hinblick auf die Reichweite von Konventionen. Konventionen werden aus der Perspektive der EC nicht lediglich für lokale Unsicherheiten zu Rate gezogen, sondern werden als Grundlage der Koordination und der Organisation von weitreichenden gesellschaftlichen Prozessen aufgefasst. Der Begriff der Konvention wird jedoch in der EC unterschiedlich gehandhabt und es wurden unterschiedliche Differenzierungen vorgeschlagen (Diaz-Bone 2016; Favereau 2008). Der gemeinsame Startpunkt stellt jedoch das Problem der kollektiven Koordination in durch Unsicherheit charakterisierten Situationen dar (Dodier 1994, S. 490). Konventionen existieren zudem aus der Perspektive der EC nur in deren pragmatischer Verwendung (Gomez 2006, S. 223). Im Folgenden werden unterschiedliche Konventionenkonzepte vorgestellt und es soll insbesondere das die Forschung anleitende Konventionenkonzept dargestellt werden. Dieses setzt sich maßgeblich aus Elementen des Konzepts der Rechtfertigungsordnung (Boltanski und Thévenot 2007), der Produktionswelten (Storper und Salais 1997), bzw. entsprechender Konventionen und den Regimen des Engagements (Thévenot 2011d) zusammen.

Die Konventionenkonzepte der EC lassen sich aufgrund von drei Dimensionen unterscheiden: Hinsichtlich ihres Miteinbezugs einer moralischen Dimension, hinsichtlich ihrer Reichweite und hinsichtlich ihrer methodologischen Verwendung in EC-basierten Arbeiten. Im deutschsprachigen Raum wird das Konventionenkonzept immer noch stark mit dem der Rechtfertigungsordnung gleichgesetzt. Wie zu zeigen sein wird, ist dieses Konventionenkonzept stark moralisiert, das zentrale Momentum dabei ist die Gleichsetzung von „Justice“ (Gerechtigkeit) und „Justesse“ (Richtigkeit), das heißt das Parallellaufen von Gerechtigkeit und Richtigkeit. Die Zuweisung von Größe hat bei Boltanski und Thévenot nicht nur eine moralische Dimension, da die Moral immer auch der Situation angepasst sein muss. Sie sprechen deswegen von einer Gleichzeitigkeit von ,justice“ und ,justesse“ (Boltanski und Thévenot 2007, S. 184). Denn was in einer Situation moralisch erscheinen mag, kann in einer anderen Situation wiederum als unmoralisch einzustufen und unpassend sein. Im Gegensatz dazu stehen stärker aus der ökonomischen Ausrichtung der EC stammende Konventionenkonzepte, welche stärker die pragmatische Unsicherheitsbewältigung durch Konventionen betonen. Die verschiedenen Konventionenkonzepte unterscheiden sich jedoch auch hinsichtlich ihrer Reichweite. Hier lässt sich wiederum zunächst auf das Konzept der Rechtfertigungsordnung verweisen, welches Konventionen mit einer sehr hohen Reichweite beschreibt, welche minimal eine Gültigkeit für viele westliche Länder beanspruchen können (Lamont und Thévenot 2000) und in der Folge werden Konventionen stärker als strukturalistische Tiefenstruktur betrachtet (Diaz-Bone 2015a; Gomez und Jones 2000). 
Dagegen existieren jedoch auch Positionen, welche einen stärker pragmatischen Einfluss haben. Dodier fasst Konventionen stärker als Handlungspotential für die situative Problembewältigung auf (Dodier 1994, 2011). Mit der Unterscheidung von Konventionen im Hinblick auf deren Reichweite hängt auch die methodologisch unterschiedliche Verwendung der verschiedenen Konventionenkonzepte zusammen. Das Konzept der Rechtfertigungsordnung wird in Studien auf Basis der EC stark als inhaltliche Heuristik an den Forschungsgegenstand herangetragen, während Konzepte mit weniger Reichweite stärker als methodologische Konzepte für ein Verständnis und eine analytische Aufarbeitung des Untersuchungsgegenstandes dienen.

Ein zentrales Konzept der EC ist das der Rechtfertigungsordnungen. Rechtfertigungsordnungen sind kollektive Handlungslogiken, welche sich historisch herausgebildet haben und eine unterschiedlich große Reichweite besitzen. In ihrem Werk „Über die Rechtfertigung“ beschreiben Boltanski und Thévenot insgesamt sechs Rechtfertigungsordnungen als fundamentale Sozio-Logiken in Frankreich. Prominent wurde eine siebte Rechtfertigungsordnung, die projektbasierte, von Luc Boltanski und Ève Chiapello in „Der neue Geist des Kapitalismus" eingeführt (Boltanski und Chiapello 2006). Die grüne Konvention ist die achte, oft referenzierte Rechtfertigungsordnung. Diese wurde von Claudette Lafaye und Laurent Thévenot eingeführt (Lafaye und Thévenot 1993) ${ }^{30}$ Rechtfertigungsordnungen sind kollektive Handlungslogiken, welche jedoch nicht intentional oder deliberativ durch eine Gemeinschaft begründet und definiert wurden (Bessy 2011, S. 179). Sie sind vielmehr das Produkt eines sozio-evolutionären Prozesses, in welchem Rechtfertigungsordnungen als kollektiv geteilte und stabilisierte Koordinationslogiken entstanden sind und ihre Wirkmächtigkeit entwickelt haben. Rechtfertigungsordnungen sind jedoch nicht an Personen, sondern an Situationen gebunden. Damit sind auch die Eigenschaften von Personen nur bis auf weiteres festgelegt, da diese Eigenschaften stets abhängig von der Perspektive der verschiedenen Rechtfertigungsordnungen sind. Rechtfertigungsordnungen sind nicht als rein diskursive Logiken gedacht. Damit ist gemeint, dass eine Rechtfertigungsordnung mit einem ganzen Ensemble an sogenannten Wesen verknüpft ist. Für Boltanski und Thévenot sind dabei Personen und Objekte gleichermaßen Wesen (Boltanski und Thévenot 2007, S. 65 f.).

\footnotetext{
${ }^{30}$ Laurent Thévenot führte außerdem die Rechtfertigungsordnung der Information ein (Thévenot 1997). James Kirwan schlug die „Regard-Convention“ vor (Kirwan 2006) und Lisa Suckert die Rechtfertigungsordnung des körperlichen Wohlbefindens (Suckert 2015, S. 196 f.). Diese Rechtfertigungsordnungen wurden bisher jedoch noch nicht breit diskutiert.
} 
Diese Verbindung ist historisch gewachsen und dadurch nicht ohne weiteres zu trennen (Dodier 2011, S. 84). Die tiefe soziokulturelle Verankerung von Rechtfertigungsordnungen im Handeln ergibt sich also nicht durch die Eigenschaft von Rechtfertigungsordnungen als reinem Diskursphänomen, sondern durch die enge Vernetzung zwischen Objekten, Handlungslogiken und Wesen. Rechtfertigungsordnungen werden folglich erst durch die Vernetzung mit Objekten zu pragmatischen Handlungswelten (Nachi 2006, S. 128). Beides, Objekte wie Wesen, funktionieren als Dispositive, welche Rechtfertigungsordnungen in Situationen auf Dauer stellen und stabilisieren. Denn der Bezug auf ein Dispositiv führt zum Bezug auf die gesamte das Dispositiv fundierende Konvention (Dodier 2011, S. 84).

Boltanski und Thévenot identifizieren insgesamt sechs Axiome, welchen gesellschaftliche Logiken entsprechen müssen, um als Rechtfertigungsordnung beschrieben werden zu können:

1. Das Prinzip des gemeinsamen Menschseins setzt eine grundsätzliche Äquivalenz zwischen den verschiedenen Personen eines Gemeinwesens voraus. Dieses erste Axiom schließt dadurch politische Modelle aus, welche die Sklaverei oder die Unterscheidung zwischen Über- und Untermenschen befürworten.

2. Das Prinzip der Verschiedenartigkeit besagt, dass sich ein Gemeinwesen durch minimal zwei unterschiedliche Merkmalszustände charakterisieren lässt. Scheinbar im Widerspruch zum ersten Prinzip stehend, wird hier folglich davon ausgegangen, dass trotz der prinzipiellen Äquivalenz zwischen den verschiedenen Mitgliedern des Gemeinwesens unterschiedliche Merkmalszustände erreicht werden können.

3. Das Prinzip der gemeinsamen Würde impliziert, dass prinzipiell sämtliche Personen Zugang zu den verschiedenen Merkmalszuständen (d. h. zu "Größe") haben müssen. Damit sind Ordnungen wie etwa das Kastensystem ausgeschlossen, da hier dauerhaft und systematisch unterschiedliche Zugangschancen zu höheren Positionen für untere Kasten bestehen.

4. Das Prinzip der Rangordnung besagt, dass sich zwischen den unterschiedlichen Merkmalszuständen eine hierarchische Rangordnung erstellen lässt. Dabei gerät dieses Axiom in einen Konflikt mit dem ersten Axiom des gemeinsamen Menschseins. Rangordnungen sind jedoch deswegen notwendig, da die höheren Merkmalszustände mit Vorteilen verknüpft sind und dadurch von einer Vielzahl von Personen angestrebt werden. 
5. Das Prinzip des Investitionsmodus besagt, dass für das Erreichen einer höheren Position Leistung investiert werden muss. Es ist dieses Prinzip, welches die Spannung zwischen den Axiomen des gemeinsamen Menschseins und den unterschiedlichen Merkmalszuständen in Einklang bringt. Das Einnehmen höherer Merkmalszustände muss gemäß diesem Axiom folglich durch Leistung gerechtfertigt sein.

6. Das Prinzip des Gemeinwohls bedeutet, dass größere Merkmalszustände mit der Zunahme eines Beitrags zum Gemeinwohl verknüpft sein müssen. Die Einnahme von höheren Positionen ist folglich an die Bedingung geknüpft, dass damit ebenfalls ein größerer Vorteil für das Gemeinwesen einhergeht.

Auf der Basis dieser durch Boltanski und Thévenot definierten Axiome wird die moralische Fundierung des Rechtfertigungskonzepts deutlich. Es baut darauf auf, dass „Größe“, d. h. ein höherer Merkmalszustand, auf Leistung beruht und einen positiven Effekt nicht nur für die „Großen“" selbst, sondern auch für das Gemeinwohl mit sich bringt. Zusätzlich zu den sechs beschriebenen Axiomen, entwickelten Boltanski und Thévenot eine Grammatik, mittels derer Rechtfertigungsordnungen systematisiert und miteinander verglichen werden können (Boltanski und Thévenot 2007, S. 196 ff.). Rechtfertigungsordnungen können anhand des übergeordneten Prinzips, das heißt dem grundlegenden Bewertungsprinzip, verglichen werden. Dies schließt eine Trennung in große (das heißt mit einer hohen Wertigkeit ausgestattet) und kleine Subjekte und Objekte ein. Damit einher geht auch der entsprechende Investitionsmodus, das heißt den Weg, den kleine Subjekte einschlagen müssen, um zu großen zu werden. Rechtfertigungsordnungen sind zudem durch typische Wesen charakterisiert. Wesen sind bei Boltanski und Thévenot Formen, welche Menschen annehmen können, wenn sie sich auf eine Rechtfertigungsordnung beziehen (Boltanski und Thévenot 2007, S. 65 f.). ${ }^{31} \mathrm{Zu}$ beachten ist dabei, dass sie sich auch auf eine Kollektivität von Personen beziehen können. Man könnte hier folglich von einer Akteursform sprechen, welche Menschen einnehmen müssen, um Wesen einer Rechtfertigungsordnung $\mathrm{zu}$ mobilisieren. Zudem werden Rechtfertigungsordnungen durch typische Objekte gestützt. Diese Stützung durch Objekte erfolgt, da Objekte viel stärker als Subjekte einzelnen Rechtfertigungsordnungen zugeteilt werden können (Thévenot 2002, S. 189). Zudem verfügt jede Rechtfertigungsordnung über

\footnotetext{
${ }^{31}$ Durch die starke Anlehnung an das Konzept der Produktionswelten ist in der empirischen Analyse nicht mehr von „Wesen“ die Rede, sondern im Hinblick auf Projektleiter von unterschiedlichen Konventionen eines Projektleiters.
} 
ein eigenes Modell der Prüfung, das heißt über ein eigenes Vorgehen, Größe festzustellen (Boltanski und Thévenot 2007, S. 196 ff.). ${ }^{32}$ Folgend werden die bereits vorgestellten acht Rechtfertigungsordnungen umfassender vorgestellt:

Im Hinblick auf die marktweltliche Rechtfertigungsordnung ist zu bemerken, dass diese vom Begriff des Marktes selber getrennt werden muss. ${ }^{33}$ Denn im alltagsweltlichen Begriff des Marktes vermischen sich in der Konzeption von Boltanski und Thévenot im Minimum die markweltliche und die industrielle Rechtfertigungsordnung (2007, S. 264) ${ }^{34}$ Zentral bei der Trennung der marktweltlichen von der industriellen Rechtfertigungsordnung ist die zeitliche Dimension: Ist die industrielle Rechtfertigungsordnung auf die langfristige Produktion ausgelegt und orientiert sich demgemäß an einem weiten zeitlichen Horizont, funktioniert die marktweltliche Rechtfertigungsordnung auf der Basis der aktuellen Nachfrage nach Gütern, was die Volatilität von Marktpreisen erklärt. Das Prinzip der marktweltlichen Rechtfertigungsordnung sind aktuelle individuelle Wünsche. Personen sind folglich durch Besitzwünsche angetrieben, was Konkurrenz begründet. Große Personen sind hier Kaufleute und reiche Personen, einen hohen Wert besitzen Produkte, welche auf dem Markt hohe Preise erzielen. Objekte sind hier durch Individuen begehrenswerte Objekte, welche verkäuflich sind. Die Wertprüfung geschieht durch ein Geschäft. Hier wird der tatsächliche Preis festgelegt, welcher der Maßstab der Größe ist (Boltanski und Thévenot 2007, S. 264 ff.).

Die industrielle Rechtfertigungsordnung ist bevölkert von wissenschaftlichen und technischen Objekten, Experten und Standards. Wichtige Prinzipien sind hier Effizienz, Leistung und Produktivität. Bewertet wird auf der Basis von Funktionalität. Es ist diese Funktionalität, welche die Eigenschaften der Gegenwart

\footnotetext{
${ }^{32}$ François Eymard-Duvernay hat das Konzept der Rechtfertigungsordnungen auch für die Analyse von unterschiedlichen Produktqualitäten in Märkten angewandt. Er spricht in diesem Kontext von Qualitätskonventionen (Eymard-Duvernay 1989). Der Einfachheit halber ist in dieser Arbeit stets von Rechtfertigungsordnungen die Rede, auch wenn eigentlich (ökonomische) Qualitätskonventionen gemeint sind.

${ }^{33}$ Zudem zeigt sich an der marktweltlichen Rechtfertigungsordnung die moralische Grundlegung der Handlungstheorie von Boltanski und Thévenot besonders deutlich. Auf einem Markt ist eigennütziges Handeln geradezu Pflicht, dies ist aus der Sicht von Boltanski und Thévenot jedoch nur möglich, weil damit ein Gemeinwohlversprechen einhergeht. Zudem wird ersichtlich, dass Markthandeln nicht als anthropologische Konstante gedacht wird, sondern dass aus der Perspektive von Boltanski und Thévenot hier bereits eine sozialisatorische Leistung vorliegt (Diaz-Bone 2009b, S. 37 f.).

${ }^{34}$ Welche von Thévenot als dominierenden Markt-Kompromissordnung der Nachkriegsjahre verstanden wird, die erst später durch die häusliche Rechtfertigungsordnung, usw. ergänzt wurde (Thévenot 1995a).
} 
in die Zukunft transportiert. Große Wesen sind professionell, sie fügen sich in ihre berufliche Funktion ein. Zentral ist in der industriellen Rechtfertigungsordnung die Plan- und Vorhersehbarkeit. Wichtige Objekte sind Instrumente (Maschinen und Werkzeuge) und die Standardisierung. Große Personen sind Experten und Spezialisten. Die Prüfung wird im Hinblick auf die Funktion durchgeführt: funktionieren die Instrumente, erfüllen Personen ihren Zweck (Boltanski und Thévenot 2007, S. $276 \mathrm{ff}$.$) ?$

Die zentralen Wesen der staatsbürgerlichen Rechtfertigungsordnung sind hier nicht einzelne Personen, sondern Kollektivpersonen. Die Vereinigung von Personen fängt bei wenigen Personen an, die größte Kollektivperson ist die Menschheit insgesamt. Groß sind Personen, welche sich dem Gemeinwillen unterordnen. Es sind folglich beispielsweise Politiker oder Gewerkschaftler, denen in dieser Rechtfertigungsordnung Größe zukommt. Es gilt hier, die Vereinzelung von Menschen zu überwinden und ein Kollektiv zu schaffen. Repräsentanten haben daher die eigenen, partikularen Wünsche dem Willen des Kollektivs unterzuordnen, was den Investitionsmodus in dieser Rechtfertigungsordnung darstellt. Zentrale Elemente sind hier die Legalität und das Mandat. Wichtige Objekte sind das Recht, die Gesetzgebung und Verfahren. Die ideale Form der Organisation ist die Demokratie. Die Prüfung erfolgt hier, indem die Wirklichkeit des Kollektivs getestet wird, das heißt insbesondere in Momenten, in welchen Gerechtigkeit durch die Berufung auf das Gesetz gefordert wird, beispielsweise in der Form von Demonstrationen oder Motionen. (Boltanski und Thévenot 2007, S. 254 ff.).

In der häuslichen Rechtfertigungsordnung ist Größe dadurch definiert, dass das Netzwerk von persönlichen Abhängigkeiten und stets nur relational bestimmbar ist. Zentrale Konzepte sind hier die Abstammung, Tradition und die Hierarchie. Dem Vater als Patron kommt eine wichtige Stellung zu, die häusliche Rechtfertigungsordnung ist patriarchalisch geprägt. Die Investition geschieht durch die Annahme einer Pflicht. Groß sind aber insbesondere auch die Ahnen und Vorfahren, da sich diese in der Generationenhierarchie an höchster Stelle befinden. Durch das Zuschreiben von Größe kommt den Personen die Pflicht zu, respektund vertrauensvoll zu handeln sowie mit Taktgefühl aufzutreten. Das gemeinsame Essen ist ein wichtiger Ort, an dem sich die Familie trifft. Objekte sind Manieren, Anstand und der Rang von Personen. Da insbesondere der Rang den Personen nicht ohne Stützen abgelesen werden kann, sind die Kleidung sowie äußere ablesbare Identifikationen wichtig, um die Frage zu ersparen, ,wer jemand ist“" Die Prüfung geschieht mittels Familienfeierlichkeiten wie Feiertagen, Geburt, Tod oder Heirat, an denen das Verhältnis der Personen zueinander aufs Neue geklärt und gefestigt wird. Eine wichtige Form der Evidenzherstellung sind in dieser Rechtfertigungsordnung Anekdoten (Boltanski und Thévenot 2007, S. 228 ff.). 
In der meinungsweltlichen Rechtfertigungsordnung wird Größe erreicht durch die Meinung anderer Personen. Große Wesen sind Personen mit hoher Bekanntheit und solche, welche allgemein geschätzt werden. Die meinungsweltliche Rechtfertigungsordnung hat dabei ein kurzes Gedächtnis: Wer heute berühmt ist, ist dies morgen vielleicht nicht mehr. Personen treten in dieser Rechtfertigungsordnung als Publikum auf, welche sich eine Meinung über jemanden bilden. Sie werden angetrieben durch eine Eigenliebe, den Wunsch berühmt zu sein. In dieser Rechtfertigungsordnung macht man sich ,einen Namen“. Objekte sind hier Marken, Nachrichten, Public Relations und Kampagnen. Die Investition erfolgt durch einen Verzicht auf das Private und das Geheimnis. Alles muss offengelegt werden. Die Kommunikation darf aber nicht zu komplex sein, da sie hier stets auf die breite Masse ausgelegt ist. In dieser Rechtfertigungsordnung wird „,beeinflusst“, die Personen „überzeugen“ einander und man achtet auf sein „Image“. Die Prüfung von Größe findet hier durch Veranstaltungen und Pressekonferenzen statt, auf denen der Ruf von jemandem zum Vorschein kommt (Boltanski und Thévenot 2007, S. 245 ff.).

Die Rechtfertigungsordnung der Inspiration ist verhältnismäßig instabil, da sie sich kaum auf äußerliche Objekte stützen kann. Inspiration kann unmöglich gemessen werden und so vollzieht sich Inspiration im Innern, fern jeglicher Standardisierbarkeit: „Wahre Größe ist ein spontaner innerer Zustand der Gewissheit, der von außen über die Wesen kommt und deshalb nicht willentlich herbeiführbar ist" (Boltanski und Thévenot 2007, S. 222). Große Wesen in dieser Rechtfertigungsordnung sind begeistert und fasziniert. Echte Inspiration kann aber auch furchterregend sein. Größe wird in dieser Rechtfertigungsordnung zudem oft nur negativ definiert, indem gängige Wege, etwas zu tun, kritisiert werden. Objekte sind hier das Unbewusste, Tagträume und allgemein Träumereien. Die Erfahrung der Inspiration betrifft dabei den Geist und Körper zugleich. Die Form der Inspiration geschieht durch den Ausbruch aus dem Gewohnten und dem Infragestellen von Etabliertem. Personen sind hier gekennzeichnet durch eine suchende Haltung. Die Prüfung findet statt durch Abenteuer, Suchen und geistige Reisen, denn dort zeigt sich, ob jemand inspiriert ist (Boltanski und Thévenot 2007, S. 222 ff.).

Die netzwerkbasierte Rechtfertigungsordnung ${ }^{35}$ wurde von Luc Boltanski und Ève Chiapello in ihrem Werk „Der neue Geist des Kapitalismus“ eingeführt

\footnotetext{
${ }^{35}$ Boltanski und Chiapello sprechen hier nicht von Rechtfertigungsordnungen, bzw. von einer Welt, sondern von der projektbasierten Polis. Das Konzept der Polis ist aber explizit an dasjenige der Rechtfertigungsordnungen angelehnt (Boltanski und Chiapello 2006, S. 149).
} 
(Boltanski und Chiapello 2006). Grundlegend ist hier das Projekt. Diese werden ins Leben gerufen, indem man aktiv Netzwerke bildet. Zentral ist in dieser Rechtfertigungsordnung die netzbildende Tätigkeit des Vermittlers. Groß ist hier, wer aktiv Netzwerke zu Projekten verknüpft. In Projekte wird man jedoch nicht eingegliedert, sondern man wählt sie aus. Es ist in der Netzwerkwelt zentral, sich selber unabhängig von Projekten weiterzuentwickeln und so seine „employability“ aufrecht zu erhalten. Das Projekt ist die zentrale Organisationsform der netzwerkbasierten Rechtfertigungsordnung, da hier eine hohe Zahl an Kontakten geknüpft wird und Projekte als flexible Antwort auf Herausforderungen erstellt werden können. Für die Mitarbeit in Projekten ist es wichtig, sich den Gegebenheiten anpassen zu können und die Mitarbeitenden begeisterungsfähig in das Projekt einzubinden. Die netzwerkbasierte Rechtfertigungsordnung sollte jedoch nicht als Rechtfertigung für egoistisches Handeln verstanden werden. Personen sind in dieser Rechtfertigungsordnung eben gerade nicht nur groß, weil sie Projekte für sich nutzen können, sondern weil sie durch dieses Engagement weitere Leute einbinden und als Teamspieler auftreten, denen die restlichen Mitarbeitenden vertrauen können (Boltanski und Chiapello 2006, S. 152 ff.).

Die grüne Rechtfertigungsordnung wurde erst im Nachgang von „Über die Rechtfertigung" beschrieben (Lafaye und Thévenot 1993). Obwohl grüne Argumentationsweisen zunehmend an Stellenwert gewinnen, taucht die grüne Rechtfertigungsordnung gemäß Thévenot et al. noch häufig in Kompromissformation mit anderen Rechtfertigungsordnungen auf (Lafaye und Thévenot 1993, S. 133). Das zentrale Prinzip ist hier, dass es zum Wohle aller Menschen ist, die Natur zu schützen (Lafaye et al. 2011, S. 157). Allerdings weisen Thévenot et al. auch darauf hin, dass weiterentwickelte Formen der grünen Rechtfertigungsordnung (die Tiefenökologie) das zentrale Axiom von Rechtfertigungsordnungen des Anthropozentrismus (Boltanski und Thévenot 2007, S. 108) hinter sich lassen und sich hin zu einem Ökozentrismus bewegen (Lafaye et al. 2011, S. 157, 163).

In Tab. 3.2 sind die acht beschriebenen Rechtfertigungsordnungen tabellarisch dargestellt. ${ }^{36}$

Auch Michael Storper und Robert Salais beziehen sich in ihrem Werk „Worlds of production“ auf das Konzept der Konvention (vgl. hierzu ausführlich Abschn. 3.2). Diese Monografie stellt dabei ein weiteres Hauptwerk der EC dar (Diaz-Bone 2018, S. 6). Das Konventionenkonzept von „Worlds of production“

\footnotetext{
${ }^{36}$ Quelle: (Boltanski und Thévenot 2007, S. 222 ff.; Lafaye und Thévenot 1993; Boltanski und Chiapello 2006).
} 


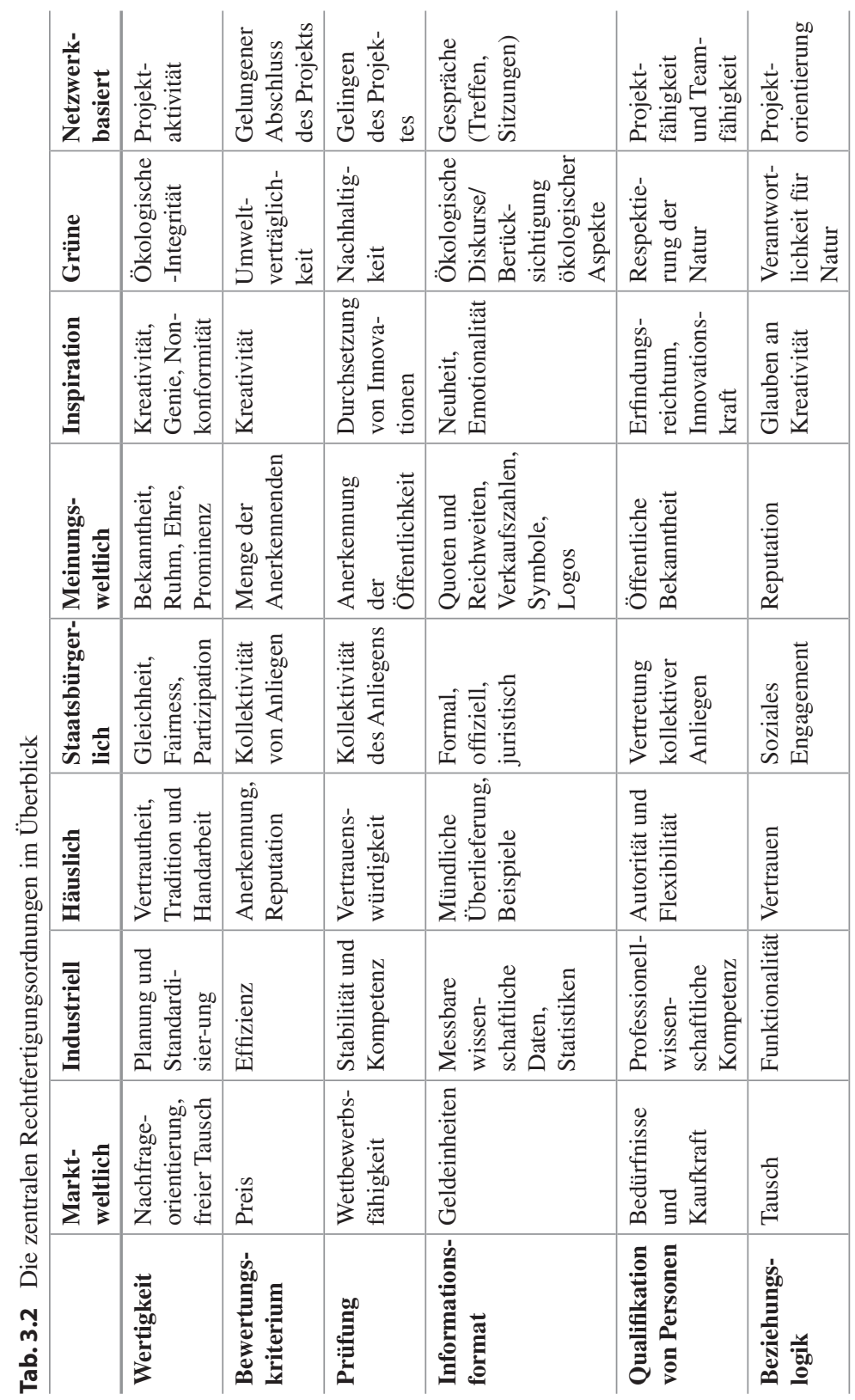


ist jedoch zu unterscheiden vom Konzept der Rechtfertigungsordnung von Boltanski und Thévenot. Denn im Gegensatz zu Boltanski und Thévenot ist es nicht das Ziel von Storper und Salais, gesamtgesellschaftliche Rechtfertigungsordnungen darzustellen, sondern vielmehr die den ökonomischen Tauschbeziehungen unterliegenden konventionellen Grundlagen. ${ }^{37}$ Sie verweisen in ihrem Konventionenkonzept auf eine frühe, spieltheoretisch inspirierte Konventionendefinition von Lewis (Storper und Salais 1997, S. 16 f.):

Eine Verhaltensregularität R von Mitgliedern einer Gruppe G, die an einer wiederholt auftretenden Situation $\mathrm{S}$ beteiligt sind, ist genau dann eine Konvention, wenn bei jedem Auftreten von $\mathrm{S}$ unter Mitgliedern von $\mathrm{G}$

(1) jeder R folgt;

(2) jeder von jedem andern erwartet, dass er R folgt;

(3) jeder es vorzieht, $\mathrm{R}$ zu folgen, sofern auch die andern es tun, weil $\mathrm{S}$ ein Koordinationsproblem ist und die allseitige Befolgung von $\mathrm{R}$ in $\mathrm{S}$ ein koordinatives Gleichgewicht ergibt (Lewis 1975, S. 43).

Entscheidend für die spezifische Verwendung dieser Konventionendefinition von Lewis ist die pragmatische Weiterführung durch Storper und Salais. ${ }^{38}$ Sie stellen die durch eine Konvention bewältigte Unsicherheitsbewältigung in den Vordergrund (Diaz-Bone 2018, S. 214). Eine solcherart verstandene Konvention reduziert folglich Unsicherheit in ökonomischen Transaktionen, da sie einen Rahmen der erwartbaren Handlungen von Anderen schafft (Storper und Salais 1997, S. 20). Zentral für das Verständnis des Konventionenkonzepts ist bei Salais und Storper das Konzept der Produktionswelten. Hier muss bereits ein wenig vorgegriffen werden. Sowohl das Konzept der Rechtfertigungsordnung als auch dasjenige der Produktionswelten stellen Weltenkonzepte dar, wenn sie auch konzeptionell unterschiedlich ausgestaltet sind. Mit Weltenkonzept ist hier gemeint, dass sowohl Rechtfertigungsordnungen, wie auch Produktionswelten, ökonomische Transaktionen umfassen, das heißt mit Bezug auf Akteure, Formen und Objekte organisieren und dadurch ermöglichen. ${ }^{39}$ Produktionswelten beziehen

\footnotetext{
${ }^{37}$ Und es ist folglich dem Konzept der Konvention bei Dodiers Untersuchungen zu den konventionellen Grundlagen der ärztlichen Diagnose näher (Dodier 1994).

${ }^{38}$ Diaz-Bone spricht von einer offeneren und vielfältigeren Anwendung des Konventionkonzepts bei Storper und Salais im Vergleich zum Konzept der Rechtfertigungsordnung (Diaz-Bone 2018, S. 212).

${ }^{39}$ Wobei die Rolle von Objekten bei der Koordination in der Produktionsweltentheorie weit weniger stark ausgebaut ist als im Konzept der Rechtfertigungsordnung von Boltanski und Thévenot.
} 
sich jedoch, wie bereits erwähnt, auf ökonomische Produktionsprozesse, während Rechtfertigungsordnungen als fundamentale gesellschaftliche Handlungslogiken verschiedenste, auch ökonomische Prozesse, anleiten können ${ }^{40}$. Zudem weisen die beiden Konzepte unterschiedliche Konstruktionsprinzipien auf. Beziehen sich Storper und Salais in ihrer Konzeptualisierung von unterschiedlichen Produktionswelten auf verschiedene Distributionsweisen von ökonomischen Transaktionen (standardisiert vs. spezialisiert und gewidmet vs. generisch) (Storper und Salais 1997, S. 26 ff.), so beziehen Boltanski und Thévenot Rechtfertigungsordnungen auf die Ideengeschichte der politischen Philosophie (Boltanski und Thévenot 2007, S. 95 ff.). Die beiden Konzepte unterscheiden sich jedoch in einem Punkt deutlich. Das Konzept der Rechtfertigungsordnung sieht vor, dass in einer ökonomischen Transaktion im Hinblick auf eine Welt sämtliche Aspekte der Transaktion auf eine die Welt anleitende Konvention bezogen werden kann. Das Konzept der Produktionswelten hingegen ist der konzeptuell integrierende Rahmen für eine Vielzahl an Konventionen. Von diesen verschiedenen Konventionen wird angenommen, dass sie miteinander zusammenhängen müssen, um einen einheitlichen Koordinationsrahmen ergeben zu können. ${ }^{41}$ Dadurch findet methodologisch einerseits eine stärkere Öffnung des Konzepts der Konvention statt. Es wird zwar ein integrativer Rahmen angenommen, in diesem Fall ist das die Produktionswelt, jedoch muss dieser im Hinblick auf viele Aspekte in eine Praxis übersetzt werden und es müssen hierzu entsprechende Konventionen etabliert werden. Produktionswelten lassen dadurch methodologisch im Vornhinein ein größeres $\mathrm{Ma} ß$ an Inkohärenzen $\mathrm{zu}$, welche selber zum Gegenstand einer Untersuchung gemacht werden können. Das Konventionenkonzept übergreift gemäß Storper und Salais drei Dimensionen: Regeln spontanen individuellen Handelns, das Erstellen von Übereinkünften zwischen Personen und Institutionen in Situationen kollektiven Handelns. Diese verschiedenen Dimensionen haben raumzeitlich unterschiedliche Reichweiten und überlappen sich in komplexer Art und Weise (Storper und Salais 1997, S. 17).

\footnotetext{
${ }^{40}$ Das Verhältnis zwischen Produktionswelten und Rechtfertigungsordnungen ist bis jetzt noch nicht geklärt (Brandl und Pernkopf-Konhäusner 2015, S. 320). Deutlich wird jedoch, dass Produktionswelten und Rechtfertigungsordnungen keine sich gegenseitig ausschließenden Konzepte sind, da deutliche Überschneidungen zwischen beiden Konzepten existieren. So weisen einige Produktionswelten starke (inhaltliche) Ähnlichkeiten mit Rechtfertigungsordnungen auf. Eine umfassende Vermittlung beider Konzepte würde jedoch den Rahmen dieser Arbeit sprengen.

${ }^{41}$ So beispielsweise der Konvention der Arbeit, der Identität und der Partizipation oder der Finanzierungskonvention (Storper und Salais 1997, S. 23; Rivaud-Danset und Salais 1992).
} 
Ein Problem, welches sich für auf der EC basierende Arbeiten ergibt, ist die fehlende Integration der verschiedenen Konventionenkonzepte. Dies wird zum Problem, wenn unterschiedliche EC-Studien, bzw. Theoretisierungen als Grundlage für die eigene Arbeit hinzugezogen werden. Klärungsbedürftig ist in einem solchen Fall, wie die verschiedenen Befunde zueinanderstehen. Diese Herausforderung stellte sich auch bei der vorliegenden Arbeit. Die grundlegende Forschungsfrage nach (unterschiedlichen) Koordinationslogiken in der Survey-Produktion war zunächst nicht fest an ein Konventionenkonzept angebunden. Einerseits wurde mit dem Konzept der Größe, bzw. Wertigkeit gearbeitet, andererseits mit demjenigen der Produktionswelten, da hier explizit transorganisationale Produktionsnetzwerke der zentrale Forschungsgegenstand sind. Das Verhältnis dieser beiden Hauptwerke kann hier zwar nicht in der notwendigen Tiefe geklärt werden, es soll jedoch mithilfe der Regimetheorie etwas Klärung zu deren Verhältnis geschaffen werden.

Die Regimetheorie von Thévenot bezieht sich direkt auf die Rechtfertigungstheorie, welche er gemeinsam mit Boltanski begründet hat. Das Rechtfertigungsregime zeichnet sich aus durch systematisierte Rechtfertigungsordnungen. Bezieht man nun die Idee der spieltheoretisch inspirierten Konventionenkonzeptualisierung (Lewis 1975; Storper und Salais 1997, S. 15 ff.) auf Regime des Engagements, so wird deutlich, dass damit ein anderes kognitives Format als bei den Rechtfertigungsordnungen abgedeckt wird. Denn die auf Lewis zurückgehende spieltheoretische Konventionenkonzeption geht gerade nicht von einer moralisch-weitreichenden Konventionenidee aus, sondern davon, dass Konventionen Antworten auf handlungspraktische Koordinationsprobleme sind (Lewis 1975, S. 6 ff.; Favereau 2008, S. 116 f.). Bezieht man diese Konventionenkonzeption auf die Regimetheorie von Thévenot, so lässt sich eine solche Konvention im Regime des planenden Handelns verorten. Es stellt sich damit jedoch die Frage, inwiefern sich auch Konventionen im planenden Handeln festlegen und definieren lassen. Favereau schafft hier Klarheit, indem er eine Auftrennung des Konventionenkonzepts vornimmt. Auf der einen Seite stehen semantisch-moralische Konventionen, auf der anderen Seite Konventionen, welche direkt Handlungsprobleme lösen (Favereau 2008). ${ }^{42}$ Folglich lässt sich auch für das Regime des planenden Handelns eine Strukturierung durch Konventionen feststellen. Diese Konventionenart lässt sich als Antwort auf handlungspraktische

\footnotetext{
${ }^{42}$ Vgl. zur Unterscheidung verschiedener Konventionenkonzeptionen in der EC auch DiazBone (2016).
} 
Koordinationsprobleme verstehen. ${ }^{43}$ Durch diesen Bezug der unterschiedlichen Konventionenkonzepte auf die Regimetheorie erfolgt ein Bruch mit einem zentralen Konzept der EC, der ,,justice et justesse“. Gerechtigkeit geht folglich nicht zwingend mit Richtigkeit einher und vice-versa. Denn es ist gerade der methodologische Fokus der Regimetheorie, Reibungen und Übersetzungsprobleme zwischen den verschiedenen Regimen aufzuzeigen (vgl. Abschn. 4.1). Deutlich wird durch diese Auftrennung des Konventionenkonzepts auf verschiedene Regime auch der unterschiedliche Fokus zwischen Rechtfertigungsordnungen und Produktionswelten. Rechtfertigungsordnungen beziehen sich auf das Rechtfertigungsregime und damit auf moralisch fundierte, weitreichende Qualitätsvorstellungen von Produkten. Im Gegensatz dazu konzentriert sich die Produktionsweltentheorie stärker auf die Organisation der Produktions- und Transaktionsprozesse und folglich auf das Regime des planenden Handelns. ${ }^{44}$

In der vorliegenden Arbeit wird konsequent zwischen Konventionen und Rechtfertigungsordnungen unterschieden. Der Begriff der Konvention wird hier als Überbegriff für soziale Logiken der Koordination verwendet, wozu auch Rechtfertigungsordnungen als Konventionen des Rechtfertigungsregimes gehören, aber auch Konventionen des Plans und des Vertrauten. ${ }^{45}$ Rechtfertigungsordnungen

\footnotetext{
${ }^{43}$ Folgt man der Idee kognitiv unterschiedlicher Konventionen weiter, so stößt man unweigerlich auf das Regime des Vertrauten. Thévenot verwendet dieses Regime auch für die Charakterisierungen von politischen Systemen (Thévenot 2014b, S. 20 f.) oder Organisationen (Thévenot 2006a, S. 131 ff.). Gefragt werden kann hier, inwiefern Koordinationsformen im Vertrautenzwischen Personen vergleichbar systematisierbar sind wie im Rechtfertigungsregime. Tatsächlich scheint es durchaus plausibel, eine gewisse Systematisierbarkeit anzunehmen. Thévenot geht davon aus, dass in jedem der verschiedenen Regime des Engagements Sozialität hergestellt werden kann. Im Regime des Vertrauten wird Sozialität durch gemeinsame Geschichten und Allgemeinplätze hergestellt, die eine „Geselligkeit“ zur Folge haben (Thévenot 2011b, S. 199 f.). Folgt man dem Gut des Regimes des Vertrauten, der persönlichen Annehmlichkeit, so lässt sich feststellen, dass sich Geselligkeit, im Sinne eines persönlichen Aufgehobenseins in einer Gemeinschaft, unterschiedlich ausgestalten kann. Einen Beitrag stellt hier die bourdieusche Idee der ,collusio“ dar, welche die sprachlich kaum zugängliche Passung zwischen Personen bezeichnet (Bourdieu 2004, S. 86). Überträgt man dieses bourdieusche Konzept auf die Theorie der Handlungsregime, wird deutlich, dass damit der Bezug auf Konventionen im Vertrauten beschrieben wird, welche sich beispielsweise zwischen Regionen und Milieus unterscheiden können.

${ }^{44}$ Produktionswelten und deren Produkte könnten folglich auch auf ihre internationale, nationale oder lokale Legitimität und Akzeptanz hin untersucht werden.

${ }^{45}$ Wobei Konventionen des Vertrauten in dieser Arbeit nicht weiter thematisiert werden, aber eine spannende zukünftige Perspektive für die Analyse von Familien-, Freundschafts-
} 
stellen aus dieser Perspektive einen Sonderfall von Konventionen dar, welche eine hohe Reichweite und so insbesondere eine hohe Relevanz für die öffentliche Koordination besitzen. In der Beschreibung der verschiedenen Survey-Welten wird primär auf produktionsweltliche Konventionen eingegangen wie diese auch von Storper und Salais eingeführt werden (Storper und Salais 1997). Die in der empirischen Ausarbeitung beschriebenen survey-weltlichen Konventionen können so primär dem Regime des Plans zugeordnet werden und stellen interpretative Grundlagen für pragmatische Handlungsprobleme dar. Dieses Konventionenkonzept steht folglich auch demjenigen von Dodier nah, welcher Konventionen ebenfalls als pragmatischen Referenzrahmen für handlungspraktische professionelle Probleme nachzeichnet (Dodier 1994). Dieser unterscheidet weiter zwischen Konventionen, welche als Common Sense einer Gesellschaft gelten und lokaleren Konventionen (Dodier 2011). ${ }^{46}$ Die im empirischen Teil der Arbeit beschriebenen survey-weltlichen Konventionen nehmen hier eine mittlere Stellung ein, da sie nicht die Reichweite von Rechtfertigungsordnungen beanspruchen können. Damit haben sie auch keinen Eingang in den gesellschaftlichen Common Sense gefunden. Andererseits gehen sie aber über lokale Konvention hinaus, da sie in einer ganzen Branche etabliert und darüber hinaus durch ihre produktionsweltliche Einbettung auf weitere Branchen übertragbar sind.

\subsubsection{Objekte, Forminvestitionen und Institutionen}

Im Hinblick auf die Rolle der Objekte in der EC zeigt sich die geteilte Position der EC zur symmetrischen Betrachtung der Akteur-Netzwerk-Theorie (Belliger und Krieger 2006). Denn Konventionen bedürfen der Abstützung durch Objekte. Erst durch diese Abstützung stabilisiert sich der Bezug auf Konventionen in einer Situation, bzw. bekommt der Bezug auf eine Konvention in einer Situation seine Legitimation. Objekte stellen Situationsevaluationen in Form von Dispositiven auf Dauer. Survey-weltliche Aushandlungen in Survey-Projekten finden folglich stets in einer ausgestatteten Umwelt statt, auf welche durch die verschiedenen Akteure als Stütze zugegriffen wird. Wie bereits dargestellt, sind Rechtfertigungs-

und Liebesbeziehungen darstellen, welche bis jetzt von der EC noch nicht aufgegriffen wurde. Konventionen des Vertrauten stellen wiederum soziale Logiken der Koordination dar, welche sich jedoch auf das Vertraute beziehen.

${ }^{46}$ Man könnte in der Folge auch von feldspezifischen Konventionen sprechen. 
ordnungen nicht lediglich sprachliche Ordnungen, sondern eng mit charakteristischen Wesen und Objekten verwoben. Während jedoch Personen in mehreren Rechtfertigungsordnungen auftreten können, sind Objekte stärker auf eine Rechtfertigungsordnung beschränkt (Thévenot 2002, S. 189). Erst durch die Vernetzung mit Objekten werden Rechtfertigungsordnungen zu pragmatischen Handlungswelten (Nachi 2006, S. 128). Es ist diese Vernetzung zwischen Objekten und Konventionen, welche dazu führt, dass beim Bezug auf ein Element automatisch das gesamte Netzwerk zum Vorschein kommt (Dodier 2011, S. 84). Die Interpretation von Situationen bedeutet dann, dass in dieser stets Wesen und Objekte vorhanden sind, welche als Argumentations- und Handlungsstützen einen Konventionenbezug fundieren können. Objekte als Dispositive determinieren dadurch nicht die Situationsevaluation, sondern stützen und stabilisieren das Engagement von Akteuren (Dodier 2011, S. 83 f.; Thévenot 2011c, S. 243). Tatsächlich sind Situationen jedoch oft durch Wesen und Objekte aus verschiedenen Rechtfertigungsordnungen bevölkert, so dass Situationsevaluationen durch Konventionen selten eindeutig sind. ${ }^{47}$ Situationen können sich folglich hinsichtlich der Klarheit ihrer Dispositiv-Ausstattung voneinander unterscheiden. Hybride Situationen bieten eher Angriffsfläche für einen Disput, während reine Situationen Personen dazu zwingen können, von einer Konvention auf eine andere umzuschwenken (Dodier 2011, S. 87).

Ein Auf-Dauer-Stellen von Koordinationsabläufen ist das Ziel der Investition in Formen. Das Konzept der Investition in Formen wurde von Laurent Thévenot und François Eymard-Duvernay eingeführt (Eymard-Duvernay und Thévenot 1983a). In seinem mittlerweile klassischen Aufsatz zur Investition in Formen macht Thévenot in direkter Anlehnung an die Idee der ökonomischen Investition die Notwendigkeit der Investition nicht nur in ökonomische Produktionsmittel deutlich, sondern auch in darauf anzupassende soziale Prozesse (Thévenot 1984). Denn mit der Anschaffung von neuen, produktiveren Maschinen ist der Transformationsprozess zu einer effizienteren Organisation noch nicht erreicht. Gleichzeitig zum Kauf von Maschinen ist es notwendig, neue organisationale Prozesse

\footnotetext{
${ }^{47}$ Der Mechanismus der Abstützung durch Objekte scheint innerhalb der EC unterschiedlich gedacht zu sein. Thévenot spricht teilweise von einer determinierenden Rolle von Objekten (Thévenot 2002, S. 193). Dodier sieht in Objekten Dispositive für das Handeln, welche nur im Extremfall eine Koordinationsform erzwingen (Dodier 2011, S. 83 ff.). Vgl. zu einer kritischen Diskussion des Objektkonzeptes Knoll (2013, S. 372 ff.). In der vorliegenden Untersuchung wird der Position von Boltanski und Thévenot gefolgt, wonach sich Dinge und Personen gegenseitig Unterstützung anbieten (Boltanski und Thévenot 2007, S. 184 f.).
} 
$\mathrm{zu}$ entwerfen und das Personal entsprechend $\mathrm{zu}$ schulen. Die Idee dahinter ist, dass konventionell fundierte Arbeitsroutinen der Abstützung durch Formen benötigen, um die prinzipielle Interpretationsoffenheit von Konventionen zu fixieren und so routinierte Handlungen im Arbeitsalltag zu ermöglichen. Formen haben folglich eine gewisse Widerständigkeit gegenüber der Interpretation. Das Argument von Thévenot ist nun, dass sich Anpassungsprozesse an neue organisationale Prozesse nicht von selbst ergeben, sondern dass dafür finanzielle und zeitliche Ressourcen aufgebracht werden müssen. Analog zu materiellen Investitionen ist es auch hier so, dass eine spätere Änderung mit Investitionskosten verbunden ist (Thévenot 1984, S. 19 f.). Formen können einerseits formeller und informeller Natur sein. Das heißt sie können als Regelung niedergeschrieben und festgelegt, oder als praktische „How-To's“ existieren. Formen unterscheiden sich zudem maßgeblich in der materialen Ausstattung, das heißt inwiefern materielle Grundlagen wie Dokumente, Formulare, aber beispielsweise auch Schalter etc. die jeweilige Form stützen. Zudem unterscheiden sie sich in Bezug auf die Reichweite ihrer Gültigkeit in zeitlicher und räumlicher Hinsicht (Thévenot 1984, S. 12). Formen kommt eine höhere Reichweite zu, wenn sie sich auf bereits existierende Formen mit hoher Reichweite stützten können, wie beispielsweise Geld oder das Recht (Thévenot 1984, S. 11, 26). Als Formen mit hoher Reichweite betrachtet Thévenot insbesondere staatliche Formen, wie beispielsweise Statistikkategorien. Das Formkonzept macht deutlich, dass Zahlen als Ergebnis verschiedener Survey-Welten eine unterschiedliche Reichweite aufweisen können, weil sie sich unterschiedlich auf Formen beziehen können, bzw. das Resultat unterschiedlicher Forminvestitionen sind.

Eine wichtige Unterscheidung ist in der EC diejenige zwischen Konventionen und Institutionen. ${ }^{48}$ Während im Neoinstitutionalismus (Dimaggio und Powell 1983; Meyer und Rowan 1977) oder in der Wissenssoziologie von Berger und Luckmann (2007) Institutionen als handlungsformend gelten, betrachte die EC Institutionen als interpretationsbedürftig (Diaz-Bone 2018, S. 371). Erst der Bezug auf eine Konvention erfüllt die Handlungsregeln einer Institution mit Handlungssinn. Institutionen werden von Akteuren also in reflexiver Weise verwendet, da sie

\footnotetext{
${ }^{48}$ Tatsächlich scheint sich das Konzept der Institution in der EC kaum vom Begriff der Form zu unterscheiden. Thévenot bezeichnet das Recht als weitreichende Form (Thévenot 1984, S. 8, 1992b, S. 1286), während Bessy und Favereau das Recht als Institution beschreiben (Bessy und Favereau 2003, S. 136 ff.). Ein Unterschied scheint lediglich in der Reichweite zu bestehen. Beiden Konzepten gemeinsam ist aus der Perspektive der EC deren Interpretationsbedürftigkeit, welche durch den Bezug auf Konventionen gelöst wird.
} 
Tab. 3.3 Mögliche Relationen von Institutionen und Konventionen

\begin{tabular}{l|l|l}
\hline \multirow{2}{*}{$\begin{array}{l}\text { Das Verhältnis von Institution } \\
\text { und Konvention(en) ist: }\end{array}$} & \multicolumn{2}{|l}{ Die Funktion einer Institution wird bewertet als: } \\
\cline { 2 - 3 } & unkritisch & kritisch \\
\hline Kohärent & 1. Normalität/Reliabilität & 2. Blockade/Hegemonie \\
\hline Inkohärent & 3. Dynamik/Wechsel & 4. Krise/Versagen \\
\hline
\end{tabular}

Quelle: (Diaz-Bone 2012, S. 71, 2017a)

Institutionen interpretativ mit Bezug auf Konventionen handhaben und anwenden. Akteure können in der EC folglich auch ohne einen Bezug auf Institutionen handeln (Salais 2003). Die Interpretationsoffenheit von Institutionen und damit einhergehenden Handlungsregeln wird in der EC jedoch nicht als Problem, sondern gerade als Lösung angesehen. Denn die Komplexität von in Regeln beschriebenen Situationen kann unmöglich vollständig in der Regel selbst spezifiziert werden. Die interpretative Auslegung löst demzufolge gerade die Unmöglichkeit der Regelspezifizierung (Favereau 1989a, S. 295). Institutionen sind in der EC als Handlungsdispositive gedacht (Diaz-Bone 2009a, S. 254 ff.). Im Unterschied zu Konventionen sind sie das Produkt intentionalen Handelns (Salais 2008b, S. 165). Konventionen und Institutionen können dadurch in verschiedenen Verhältnissen zueinander existieren. ${ }^{49}$ Diaz-Bone beschreibt anhand von zwei Dimensionen vier mögliche Relationen, welche in Tab. 3.3 dargestellt sind.

In der ersten Situation wird das Funktionieren der Institution als unkritisch bewertet und die pragmatische Anwendung der Institution gelingt ohne Probleme, da die Institution kohärent mit der beigezogenen Konvention, bzw. der Rechtfertigungsordnung, ist. In der zweiten Situation besteht ebenfalls ein kohärentes Verhältnis zwischen Institution und Konvention, d. h. die Institution kann sinnvoll angewendet werden. Jedoch wird die Funktion der Institution als kritisch empfunden, d. h. sie wird auf Basis des Gemeinwohls kritisiert. Kritik ist in dieser Situation jedoch schwierig, da das Funktionieren der Institution nicht in Widerspruch zur beigezogenen Konvention steht. In der dritten Situation wird die Institution als unkritisch empfunden, steht jedoch in einem Widerspruch zur beigezogenen Konvention. Dadurch wird aus Sicht der Akteure ein Wechsel $\mathrm{zu}$ anderen, die Institution fundierenden Konventionen angestrebt, was eine

\footnotetext{
${ }^{49}$ Und es kann argumentiert werden, dass Institutionen in den in der folgenden Abbildung dargestellten Situationen 2, 3 und 4 exogen und in der Situation 1 endogen funktionieren (Diaz-Bone 2009a, S. 256).
} 
Dynamik der konventionellen Grundlage zur Folge hat. In Situation vier wird sowohl das Funktionieren der Institution kritisiert und es existiert gleichzeitig eine Inkohärenz zwischen Institution und Konvention. Dadurch wird, wie bereits in Situation zwei, Kritik an der Institution formuliert, welche hier jedoch erfolgreicher ist als in der zweiten Situation, da das Verhältnis zwischen Institution und Konvention selbst als kritisch empfunden wird (Diaz-Bone 2012, S. 71 f.). Mit der Unterscheidung zwischen Konventionen und Institutionen und der Beschreibung von verschiedenen Relationen zwischen beiden Konzepten eröffnet sich eine Perspektive auf das Verhältnis von Institutionen, Standards und Regeln und deren situationsspezifischer Interpretation. Für die vorliegende Arbeit ist dies insbesondere mit Blick auf die Etablierung von Qualitätskriterien für die Survey-Forschung von Bedeutung. Denn hier findet gerade eine Investition in Formen, bzw. eine Institutionalisierung von Qualität auf der Basis von Konventionen statt, deren Formen in anderen Situationen dysfunktional sein können, da sie nicht sinnvoll auf andere Konventionen der Survey-Qualität bezogen werden können.

\subsubsection{Dispute und Kompromisse}

Wie bereits im Kapitel zu Objekten, Forminvestitionen und Institutionen deutlich wurde, thematisiert die EC maßgeblich die Herstellung einer kollektiven Einigung durch Akteure in ausgestatteten Umwelten. Im folgenden Unterkapitel wird nun spezifischer auf Dispute und Kompromisse eingegangen. Dispute und Kompromisse sind im Hinblick auf Survey-Welten zentrale Elemente der Analyse von Aushandlungen im Hinblick auf Fragen der Survey-Qualität und der Arbeitsorganisation.

Boltanski und Thévenot interessieren sich in „Über die Rechtfertigung“ für Situationen, in denen versucht wird, Kontingenzen zu bewältigen und eine allgemeine Ordnung in einer Situation herzustellen (Boltanski und Thévenot 2007, S. 181). Dieses Erreichen von Allgemeinheit bedarf jedoch der Allgemeinheitsfindung. Sie führen dazu das Konzept der Prüfung ein. Bei der Prüfung geht es nicht darum, dass Akteure individuelle Prüfungen vornehmen. Gefragt ist demnach nicht, was persönliche Meinungen an Größe zuordnen, sondern was allgemein anerkannte Größen der Personen und Objekte in der entsprechenden Situation sind. Eine Prüfung enthält zwei Schritte: Einerseits muss geklärt werden, welche Konvention legitimerweise als Bewertungsgrundlage hinzugezogen werden soll und andererseits, welche die sich daraus ergebenden Größen der Personen und Objekte sind. In beiden Schritten nehmen gemäß Boltanski und 
Tab. 3.4 Kritik zwischen Rechtfertigungsordnungen

\begin{tabular}{l|l|l|l}
\hline & \multicolumn{3}{|c}{ Akteur } \\
\hline \multirow{2}{*}{ Interpretation } & Staatsbürgerlich & Staatsbürgerlich & Markt \\
\cline { 2 - 4 } & Markt & Naiv & Gierig \\
\hline
\end{tabular}

Quelle: Thévenot (2002, S. 6)

Thévenot Objekte eine zentrale Koordinationsfunktion ein (Boltanski und Thévenot 2007, S. 185). ${ }^{50}$ Die Verknüpfung von Rechtfertigungsordnungen und Dingen „erlöst“ die in einen Disput geratenen Akteure von der absoluten Deutungskontingenz einer Situation. Zwischen Rechtfertigungsordnungen können typische Kritiken formuliert werden. Thévenot macht dies anhand der staatsbürgerlichen und der marktweltlichen Rechtfertigungsordnung deutlich. Die verschiedenen Relationen werden in Tab. 3.4 dargestellt.

Durch die Kreuztabellierung werden einerseits Passungsverhältnisse, aber auch Kritiken der beiden Rechtfertigungsordnungen untereinander sichtbar. „Realistisch“ sind Personen aus der Perspektive der marktweltlichen Rechtfertigungsordnung dann, wenn sie die Konsumentenabhängigkeit sowie die temporal starken Marktschwankungen einbeziehen. Hingegen erscheint der Bezug auf die staatsbürgerliche Rechtfertigungsordnung aus der Perspektive der marktweltlichen Rechtfertigungsordnung als ,naiv“. Denn die marktweltliche Rechtfertigungsordnung geht davon aus, dass Menschen durch individuelle Wünsche (und entsprechende Nutzenmaximierung) angetrieben sind. Der „Glaube“ der staatsbürgerlichen Rechtfertigungsordnung an das Kollektiv ist aus dieser Perspektive naiv, da aus Sicht der marktweltlichen Rechtfertigungsordnung die kollektive Orientierung von Akteuren überschätzt wird. Umgekehrt ist die Orientierung an der marktweltlichen Rechtfertigungsordnung aus der Perspektive der staatsbürgerlichen Rechtfertigungsordnung ,gierig“, da hier maßgeblich aus Eigeninteresse gehandelt wird und dadurch keine Orientierung am Kollektiv stattfindet. Sichtbar wird in dieser Tabelle, dass Kritik aus der Perspektive

\footnotetext{
${ }^{50}$ Boltanski und Thévenot erwähnen, dass Personen und Dinge sich wechselseitig ihre Unterstützung anbieten. Halten beide Seiten zusammen, dann liefere dies den Beweis dafür, „dass Einigungen zwischen Menschen gerecht sind“ (Boltanski und Thévenot 2007, S. 184 f.). Sie sprechen in der Folge auch von einer Objektivierung durch Dinge.
} 
der EC nicht „,neutral“, sondern nur durch den Bezug auf eine Rechtfertigungsordnung formuliert werden kann. Die Kreuztabellierung ermöglicht die systematische Analyse von Passungs-, Kritik- und Konfliktverhältnissen zwischen verschiedenen Handlungslogiken und forciert so eine systematische Analyse von Relationen zwischen unterschiedlichen Konventionen.

Eine Prüfung der Situation wird immer dann notwendig, wenn die Deutung der Größe von Objekten und Personen zwischen Personen divergiert. Dies führt zu einem Missklang in Situationen, was der Klärung bedarf. Tatsächlich ist gemäß Boltanski und Thévenot keine Situation auf Dauer völlig klar. Trotz des Dispositivkonzeptes kommt es auf die Akteure an, Ordnung herzustellen. Eine Prüfung geschieht in jeder Konvention durch eigene kognitive Formen (Boltanski und Thévenot 2007, S. 186): Während in der industriellen Rechtfertigungsordnung Größe in Form von Instrumenten und Standards ,gemessen“ wird, geschieht die Klärung von Größe in der Rechtfertigungsordnung des Hauses durch die Offenlegung der (familiären) Herkunft. Die grüne Konvention schlussendlich ist beispielsweise auf die industrielle Konvention angewiesen, um den biologischen Fußabdruck messen und kommunizieren $\mathrm{zu}$ können (Diaz-Bone 2018, S. 157). Damit wird deutlich, dass ,Survey-Qualität“ und auch die Qualität der Zusammenarbeit mit einer Survey-Firma in den verschiedenen Survey-Welten nicht lediglich unterschiedlich definiert, sondern auch auf unterschiedliche Arten evaluiert wird.

Gerade in den Eigenheiten eines Disputes zeigt sich das Beherrschen von mehreren Rechtfertigungsordnungen. Ein Disput kann dabei als Streit darüber gedacht werden, welche Konvention als Äquivalenzprinzip zur Anwendung kommen soll. Dies bedingt jedoch der Kenntnis der Bezugskonvention des Gegenübers (Dodier 2011, S. 81). Akteure sind damit selbst in Disputen durch die gemeinsame Wissensbasis verbunden und unterscheiden sich lediglich bei der Frage der Anwendung. Ein Disput kann gemäß Boltanski und Thévenot vier mögliche Konsequenzen mit sich ziehen: Beilegung des Disputs durch den Bezug auf eine Rechtfertigungsordnung, Weiterführung des Disputs, Relativierung des Disputs durch den Wechsel in ein anderes Handlungsregimes ${ }^{51}$ oder die Erstellung eines Kompromisses. Einen Kompromiss zu erstellen bedeutet, ein hybrides Setting zu produzieren, welches nicht eindeutig einer Konvention zuweisbar ist (Boltanski und Thévenot 2007, S. 367 ff.). Ein Kompromiss

\footnotetext{
${ }^{51}$ Vgl. für einen Überblick zur vielfältigen Figur des Regimewechsels Knoll (2013, S. 374 ff.).
} 
versucht, einen Brückenschlag zwischen Konventionen herzustellen und so einen Mittelweg zu gehen. Kompromisse sind dadurch aber auch angreifbar. Denn der Bezug auf die eine Konvention eröffnet die fehlende Bezugnahme auf die jeweils andere Rechtfertigungsordnung. Um einen Kompromiss stabiler und dauerhafter machen zu können, ist es notwendig, diesen durch „hybride Objekte“ und „mehrdeutige Wesen“ zu stützen. Objekte, welche in allen beteiligten Rechtfertigungsordnungen Größe innehaben, werden so als Stabilisatoren eingesetzt, um die parallele Koexistenz mehrerer Rechtfertigungsordnungen unter Beweis zu stellen. $^{52}$

Ein zentrales Thema der Soziologie der Konventionen stellen unterschiedliche Wertigkeiten von Objekten und Personen dar. Diese sind abhängig von der evaluierenden Konvention. Der Prozess der Zuschreibung von Wertigkeit wird in dem Werk „Experts et faussaires“ von Christian Bessy und Francis Chateauraynaud thematisiert. Mit dem Konzept der „Prise“ bezeichnen sie die Evaluation und Valuation von Wertigkeit (Bessy und Chateauraynaud 2014, S. 291 ff.). In „Über die Rechtfertigung“ wird diese Wertigkeitszuschreibung nicht weiter thematisiert. Die Zuschreibung von Wertigkeit erfolgt folglich „problemlos“ durch den Bezug von Objekten und Personen auf eine Konvention. Bessy und Cheateauraynaud hingegen unterscheiden verschiedene Dimensionen der Prüfung von Wertigkeit (Bessy und Chateauraynaud 2014, S. 300). Nebst der doppelten Komplexität von Koordination aus Sicht der EC durch die horizontale Differenzierung in unterschiedliche Rechtfertigungsordnungen und die vertikale Differenzierung in verschiedene Handlungsregime wird durch die Arbeit von Bessy und Chateauraynaud zusätzlich eine doppelte Unsicherheit eingeführt. Die erste Unsicherheit ergibt sich dabei aus der von Boltanski und Thévenot beschriebenen doppelten Komplexität (Boltanski und Thévenot 2007; Thévenot 2011d). Objektgestützt gilt es hier zu klären, in welcher Situation - im Hinblick auf Regime und Rechtfertigungsordnungen - man sich befindet. Die von Bessy und Chateauraynaud eingeführte zweite Unsicherheit besteht darin, dass die Objektstützung selbst eine Quelle der Unsicherheit darstellen kann, so dass diese gegebenenfalls weiter geklärt und so Unsicherheit minimiert werden muss. Aus der Perspektive von Bessy und Chateaureynaud wird folglich deutlich, dass sich Akteure in Survey-Welten bei der Evaluation von Survey-Qualität nicht blind auf Objekte und Formen verlassen können, sondern auch diese Stützen selbst zum

\footnotetext{
${ }^{52}$ Boltanski und Thévenot nennen beispielsweise „Autorität“ und „Verantwortlichkeit“ als mehrdeutige Wesen, welche sowohl in der häuslichen als auch in der industriellen Konvention Größe beanspruchen können (Boltanski und Thévenot 2007, S. 371).
} 
Gegenstand einer kritischen Qualitätsevaluation machen müssen. Projektleiter in Survey-Projekten müssen folglich stets über das Unmittelbare hinausgehen und beispielsweise die Herkunft und spezifische Verwendung von Objekten und Formen mit in ihre Evaluation einbeziehen.

\subsubsection{Der reflexive Akteur}

An der Einführung des Konzepts des reflexiven Akteurs wird die direkte Absetzung von der bourdieuschen Soziologie deutlich. Denn die Proklamation eines reflexiven Akteurs ist erst dann notwendig, wenn zuvor von wenig reflektierten Akteuren ausgegangen wurde. Im Gegensatz zur Habitus- und Dispositionentheorie Bourdieus (1993, S. 97 ff.) betrachtet die EC Akteure nicht als durch einen inkorporierten Habitus angetrieben an, welcher soziale Praktiken auf Dauer stellt. Handlung findet aus der Perspektive der EC folglich nicht durch den Bezug auf ein (klassenabhängiges) sozialisiertes und fixiertes Ich statt, sondern vielmehr reflexiv und distribuiert durch den situativ unterschiedlichen Bezug auf Konventionen, Formen und Objekte. Die unterschiedliche Bewertung von Survey-Qualität in den verschiedenen Survey-Welten lässt sich folglich nicht alleine auf einen unterschiedlichen Sozialisierungshintergrund der Akteure beziehen, sondern muss vielmehr in einer kompetenten Situationsdefinition der Akteure selbst gesucht werden. Entsprechend sollte „Survey-Qualität“ nicht auf unterschiedliche Akteursgruppen bezogen werden, sondern auf unterschiedliche Umwelten, in welchen Akteure Survey-Qualität situativ kompetent bewerten.

Der reflexive Akteur ist so in der EC einerseits als fähiger, andererseits auch als unvollständiger Akteur konzipiert. Er ist insofern fähig, da er Situationen mit Bezug auf Konventionen, Formen und Objekte gekonnt meistern und lösen kann, was als Arrangement-Fähigkeit beschrieben werden kann. Diese Arrangement-Fähigkeit umfasst das Formulieren von guten Argumenten und das Herstellen von dazu passenden objekthaften Arrangements (Boltanski und Thévenot 2007, S. 307 ff.; Boltanski 2012, S. 38). Andererseits ist er unfähig, da er für die Kontingenzüberwindung auf Objekte und Konventionen als Stützen angewiesen ist. Der Akteur ist in der EC nicht als Einheit mit einer stabilen Identität gedacht (Dodier 2011, S. 88; Thévenot 2009a, S. 52), sondern multipel und vielfältig. Hier kristallisiert sich quasi die von der EC auf verschiedene Weise vorgeschlagene doppelte Pluralität heraus. Das Konzept der doppelten Pluralität weist einerseits daraufhin, dass unterschiedliche Sozio-Logiken in Form von Rechtfertigungsordnungen parallel nebeneinanderstehen und virtuell immer verfügbar sind (Diaz-Bone 2011a, S. 23). Verdoppelt wird diese Pluralität durch das 
Hinzukommen unterschiedlicher Regime des Engagements (vgl. Abschn. 3.1.1), unterschiedlicher Handlungsregime (Boltanski 2012) und durch unterschiedliche Konventionenkonzepte (Favereau 2008). ${ }^{53}$ Akteure verfügen folglich grundsätzlich nicht über eine situationsübergreifende einheitliche Identität. Mit Boltanski und Thévenot lässt sich vielmehr formulieren, dass sie in unterschiedlichen Situationen zu unterschiedlichen Wesen werden müssen. Dies kommt daher, da unterschiedliche Rechtfertigungsordnungen durch unterschiedliche Wesen charakterisiert sind. Wesen bezeichnen bei Boltanski und Thévenot sowohl Personen wie auch Objekte, welche den Bezug auf Rechtfertigungsordnungen stützen können (Boltanski und Thévenot 2007). Durch den Bezug auf eine Rechtfertigungsordnung muss auch in die Rolle eines solchen Wesens geschlüpft werden, um als qualifizierter Akteur auftreten zu können. Das Konzept der Wesen lässt sich deswegen als Akteursform interpretieren. ${ }^{54}$

Das Akteurskonzept der EC distanziert sich von utilitaristischen Akteurskonzeptionen, da diese den Nutzen an den Beginn der handlungstheoretischen Überlegungen setzen. Der Akteur der EC ist hingegen getrieben durch den Willen der gesellschaftlichen Teilhabe an einer gemeinsamen Sache. Thévenot spricht hierbei von „Engagement" in einer gemeinsamen Sache (französisch „chose commune“) (Thévenot 2011d, S. 259). Damit eine solche Teilhabe überhaupt möglich wird, gilt es die Situation zu klären. Zuerst müssen deshalb Kontingenzen überwunden werden (Boltanski und Thévenot 2007, S. 181). Durch den Bezug auf eine Konvention werden solche Kontingenzen überwunden und Handlungspotenzial freigesetzt. Ein grundlegendes Problem für den Akteur ist in der EC die Vielzahl der abrufbaren Konventionen. Denn Konventionen sind als (virtuelle) Handlungslogiken ständig für die Akteure verfügbar (Diaz-Bone 2011a, S. 23). Die Entscheidung für den Bezug auf eine Konvention geht deswegen mit dem Ignorieren der restlichen - ebenfalls potenziell verfügbaren - Konventionen einher. Diesen Mechanismus nennen Boltanski und Thévenot geöffnete und geschlossene Augen (Boltanski und Thévenot 2007, S. 313 f.; Thévenot 2011e, S. 36), da das Engagement für eine relevante Konvention gleichzeitig das Ignorieren der anderen

\footnotetext{
${ }^{53}$ Leider sind die verschiedenen Konzepte der zweiten Komplexität bis jetzt nicht aufeinander bezogen worden. So müsste eigentlich von einer dreifachen Komplexität die Rede sein, da beispielsweise die verschiedenen Regime des Engagements bei Thévenot nicht deckungsgleich mit den Handlungsregimen bei Boltanski sind und so eine dritte Komplexität in die EC-Analyse einbringen.

${ }^{54}$ In einer strukturalistischen Betrachtung weist das Konzept der personalen Wesen, bzw. der Akteursform, Ähnlichkeiten mit dem Konzept der Subjektposition auf (Foucault 2008, S. 528 ff.).
} 
Konventionen bedeutet. ${ }^{55}$ Für eine gelungene Koordination sind sowohl geöffnete als auch geschlossene Augen unumgänglich. Denn auch geschlossene Augen sind notwendig, um in Situationen eine gemeinsame und einheitliche Koordinationsbasis zu erreichen.

Die Absetzung von der bourdieuschen Akteurskonzeption erschöpft sich jedoch nicht mit dem Hinweis auf den reflexiven Akteur und dessen Pluralität. Die EC kann als methodologischer Situationalismus aufgefasst werden (vgl. zur Diskussion der methodologischen Grundlegung der EC Abschn. 4.1). In der Folge findet eine Dezentrierung von Agency statt, welche nun als das Produkt einer Ko-Konstruktion der verschiedenen Entitäten einer Situation wie Objekte, Formen, Konventionen und Akteure aufgefasst wird (Diaz-Bone 2011a, S. 27). Der Situationsbegriff der EC darf dabei nicht mit face-to-face Situationen gleichgesetzt werden. Er wird in der EC sehr weit gefasst und beschreibt „,komplexe Konstellationen von Objekten, kognitiven Formaten, Koordinationserfordernissen (Probleme), institutionellen Arrangements (wie Organisationen), Personen und Konzepten“ (Diaz-Bone 2018, S. 374 ff.). Situationen können in der EC dementsprechend auch raumzeitlich weit ausgedehnte Konstellationen darstellen (Diaz-Bone 2018, S. 374 ff.).

\subsection{Produktionswelten}

The precondition of coordination is that participants agree as to the qualities of products bought or sold. In the absence of such an agreement, the exchange cannot take place. By „qualities“ we do not mean „good“ or „bad“ quality, but the fact that any exchange presupposes an agreement about what the product is (Storper und Salais 1997, S. 38).

Ein für die EC zentrales Konzept ist dasjenige der Produktionswelten. „Worlds of Production“ von Michael Storper und Robert Salais stellt das zweite Hauptwerk der EC neben „Über die Rechtfertigung“ dar (Diaz-Bone 2018, S. 32). Im Zentrum stehen hier Fragen nach der Entwicklung von wirtschaftlichen Clustern. Storper und Salais können aufzeigen, wie unterschiedlich die Produktionsweisen in verschiedenen Wirtschaftsclustern organisiert sind und auf welchen unterschiedlichen Konventionen die verschiedenen „Produktionswelten“ basieren. Sie folgen damit Arbeiten von Michael Piore und Charles Sabel, welche die Idee der industriellen Effizienzsteigerung als einzige Form wirtschaftlicher Entwicklung kritisiert haben

\footnotetext{
${ }^{55}$ Boltanski spricht vom hermeneutischen Widerspruch und von der Ambivalenz von Institutionen (Boltanski 2008, S. 136).
} 
(Piore und Sabel 1989). Das Konzept der Produktionswelten ist die zentrale Referenz für die später dargestellten Survey-Welten. Es ermöglicht eine umfassende Theoretisierung von Survey-Qualität und der unterschiedlichen Produktions- und Koordinationsprozesse in den verschiedenen Survey-Welten. Zentral ist dabei die Unterscheidung zwischen „möglichen“ und „tatsächlichen“ Produktionswelten (Storper und Salais 1997, S. 97 ff.). „Mögliche“ Produktionswelten stellen idealtypische Produktionswelten dar, wie sie in der Praxis kaum anzutreffen sind. Denn aus der Perspektive von Storper und Salais stellen „tatsächliche“, d. h. real anzutreffende Produktionswelten meistens einen Kompromiss aus verschiedenen „möglichen“ Produktionswelten dar (Storper und Salais 1997, S. 77 ff.).

\subsubsection{Produktionswelten als Koordinationsrahmen der Ökonomie}

Anhand von zwei Grunddimensionen entwerfen Storper und Salais vier mögliche, d. h. idealtypische Produktionswelten. Idealtypen sind jedoch nicht nur eine wissenschaftliche Konstruktion, sondern begründen auch für die in einer Produktionswelt involvierten Akteure die pragmatische Handlungsreferenz (Storper und Salais 1997, S. 27). Eine zentrale Herausforderung von Produktionswelten ist folglich auch die Ausrichtung von individuellen Handlungen an den grundlegenden Konventionen einer Produktionswelt (Storper und Salais 1997, S. 41). Die erste Dimension unterscheidet die ökonomische Ausrichtung zwischen standardisierten und nicht standardisierten Produkten. Die zweite Dimension unterscheidet zwischen gewidmeten (,dedicated“) und generischen (,generic“) Produkten. Dadurch ergibt sich eine Aufteilung von ökonomischen Koordinationsprozessen in vier Felder.

Die von Storper und Salais beschriebenen Produktionswelten sind die industrielle Welt, die Marktwelt, die interpersonelle Welt und die Welt der intellektuellen Ressourcen. Sie sind einerseits gekennzeichnet durch unterschiedliche Formen der Produkt- und Qualitätsevaluation, der Unsicherheitsdefinition und -wahrnehmung sowie der Grundlage des Wettbewerbs als auch - wie noch zu zeigen sein wird - durch unterschiedliche Konventionen der Arbeit sowie der Finanzierung. Produktionswelten sind dadurch nicht mit einer Konvention gleichzusetzen, sondern durch mehrere Konventionen fundiert. Dabei wendet sich das Produktionsweltenkonzept gegen eine Trennung zwischen Produkten und Dienstleistungen, da aus der Produktionsweltenperspektive jedes Produkt auch eine Dienstleistung beinhaltet (Storper und Salais 1997, S. 39). In Tab. 3.5 sind die möglichen Produktionswelten gemäß Storper und Salais dargestellt. 
Tab. 3.5 Mögliche Produktionswelten

\begin{tabular}{|c|c|c|}
\hline & $\begin{array}{l}\text { Spezialisierte Produkte } \\
\text { (economies of variety) }\end{array}$ & $\begin{array}{l}\text { Standardisierte Produkte } \\
\text { (economies of scale) }\end{array}$ \\
\hline $\begin{array}{l}\text { Gewidmete Produkte } \\
\text { (Unsicherheit im Sinne von } \\
\text { Knight) }\end{array}$ & Interpersonelle Welt & Marktwelt \\
\hline Qualitätsevaluation: & Preis & $\begin{array}{l}\text { Industrielle Standards aus Sicht } \\
\text { der Nachfrager }\end{array}$ \\
\hline Unsicherheit: & $\begin{array}{l}\text { Persönliche Eigenschaften } \\
\text { anderer Produzenten und } \\
\text { Konsumenten }\end{array}$ & $\begin{array}{l}\text { Veränderliche Preise und } \\
\text { Mengen }\end{array}$ \\
\hline Wettbewerbsgrundlage: & $\begin{array}{l}\text { Verständigung innerhalb } \\
\text { einer Gemeinschaft von } \\
\text { Personen } \\
\text { Qualität }\end{array}$ & Preise und Liefergeschwindigkeit \\
\hline $\begin{array}{l}\text { Generische Produkte } \\
\text { (Risiko im Sinne von } \\
\text { Knight) }\end{array}$ & $\begin{array}{l}\text { Welt der intellektuellen } \\
\text { Ressourcen }\end{array}$ & Industrielle Welt \\
\hline Qualitätsevaluation: & $\begin{array}{l}\text { Wissenschaftliche } \\
\text { Methoden }\end{array}$ & $\begin{array}{l}\text { Allgemeine industrielle } \\
\text { Standards }\end{array}$ \\
\hline Unsicherheit: & $\begin{array}{l}\text { Pfad der } \\
\text { Wissensentwicklung }\end{array}$ & $\begin{array}{l}\text { Business cycle, } \\
\text { Nachfrageschwankungen }\end{array}$ \\
\hline Unsicherheitsbewältigung: & Vertrauen in Andere & $\begin{array}{l}\text { Kurz- und mittelfristige } \\
\text { Vorausplanung }\end{array}$ \\
\hline Wettbewerbsgrundlage: & Lernen & Preis \\
\hline
\end{tabular}

Quelle: (Storper und Salais 1997, S. 33)

Die industrielle Welt funktioniert über allgemeine, standardisierte Produktqualitäten. Die jeweilige Qualität ist entsprechend sämtlichen Marktakteuren bewusst und dadurch vergleichbar. Die Produktion ist langfristig geplant und nicht an spezifischen Käufern ausgerichtet, sondern an einem Massenmarkt. Durch die grundlegende Substituierbarkeit der in der industriellen Welt produzierten Produkte funktioniert der Wettbewerb über den Preis. Ein realer Idealtyp der industriellen Welt ist die Einführung der tayloristischen Produktionsweise.

Die Marktwelt basiert auch auf standardisierten Produkten, der Zeithorizont ist jedoch kürzer und auf eine aktuelle Nachfrage der Konsumenten ausgelegt. Die Orientierung am aktuellen Marktgeschehen führt zu Anpassungsbestrebungen an aktuelle Preis und Nachfrageentwicklungen. Im Gegensatz zur interpersonellen Welt besteht hier ein sichtbares Angebot, jedoch ist dieses an den wandelnden Bedürfnissen der Konsumenten ausgelegt. Durch diese schwankenden Bedürfnisse findet der Wettbewerb nicht nur um den Preis statt, wie in der industriellen 
Welt, sondern zusätzlich auch über die Verfügbarkeit von den Konsumentenwünschen entsprechenden Produkten.

Die interpersonelle Welt funktioniert über eine individualisierte Produktion. Die Produkte werden nicht für einen Massenmarkt, sondern auf bestimmte Klientelgruppen oder gar Einzelkunden hin produziert. Der Wettbewerbsmechanismus ist dadurch nicht der Preis - die individualisierte Produktion wäre hier unmöglich wettbewerbsfähig - sondern die individualisierte Qualität. Qualität wird in dieser Welt gemäß Storper und Salais über den Preis von Produkten evaluiert (Storper und Salais 1997, S. 33) ${ }^{56}$ Die sich daraus ergebende, von außen schwierig einzuschätzende Qualität der jeweiligen Produkte stellt auch an den Käufer große Ansprüche, da dieser Wissen mitbringen muss, um die jeweilige Qualität bewerten zu können. Die Produktion der Güter geschieht in engmaschigen Netzwerken zwischen Käufern und Produzenten, welche oft auf wiederholten Markthandlungen basieren.

Die Welt der intellektuellen Ressourcen ist wissensintensiv und wissensbasiert. Im Gegensatz zur interpersonellen Welt wird hier nicht auf eine spezifische Klientelgruppe hin produziert, sondern wiederum für einen Massenmarkt. Im Unterschied zur Marktwelt ist die Produktion nicht an einer aktuellen Nachfrage orientiert. Denn durch das Einführen beispielsweise neuer technischer Standards oder Plattformen werden Bedürfnisse erst geschaffen. Hier werden neue Standards gesetzt, beispielsweise durch die Etablierung von neuen Softwareprogrammen, neuen Verschlüsselungstechniken oder neuen Technikstandards. Beispiele für diese Produktionswelt sehen Storper und Salais in wissensintensiven Entwicklungsprojekten von Firmen, auch solchen, welche grundsätzlich in der Marktwelt oder industriellen Welt tätig sind.

Die verschiedenen Produktionswelten sind durch unterschiedliche Arten der Qualitätsunsicherheit auf Käuferseite gekennzeichnet. Die industrielle Welt ist durch eine relativ kleine Unsicherheit bezüglich der eingekauften Produktqualität gekennzeichnet, da die verschiedenen Produzenten eine standardisierte und

\footnotetext{
${ }^{56}$ Der Preis als Basis von Qualitätsevaluationen in der interpersonellen Welt erscheint zunächst nicht intuitiv, gerade auch weil diese Welt im Hinblick auf die Preise der in dieser Welt hergestellten Produkte nicht konkurrenzfähig ist mit anderen Welten. Die von Storper und Salais beschriebene Form der Unsicherheit stellt in dieser Welt die Kompetenz der Produzenten dar (1997, S. 33). Ist diese Unsicherheit überwunden, wird deutlich, dass mit mehr Arbeitseinsatz auch eine höhere Qualität des entsprechenden Produkts entsteht, bedingt durch die maßgeschneiderte Produktionsweise in dieser Welt, welche keine Economies of Scale ermöglicht. Entsprechend steht - nach Überwindung der genannten Unsicherheiten - der Preis für die Qualität des Produktes.
} 
generische und somit vergleichbare Qualität herstellen. Die Qualität ist hier mit minimalen Schwankungen bereits im Voraus bestimmbar. Im Gegensatz dazu ist eine Qualitätsbestimmung in der interpersonellen Welt vor dem Kauf durch die unstandardisierte und spezialisierte Produktionsweise unmöglich. Die Qualität ist hier erst nach dem Kauf möglich, da erst dann das eigentliche Produkt identifizierbar wird (Storper und Salais 1997, S. 31 ff.). Daraus folgt, dass den Käufern in den verschiedenen Produktionswelten ein unterschiedlicher Grad an Mündigkeit im Hinblick auf das Marktangebot zukommt. Der eine Idealfall besteht wiederum in der industriellen Welt. Durch die standardisierten und generisch sichtbaren Standards der Produktion kommt dem Konsument hier eine hohe Mündigkeit im Hinblick auf das Bewerten des Marktangebotes zu. Der Konsument entspricht in dieser Welt damit am ehesten dem Idealbild des informierten und rationalen Käufers der neoklassischen Wirtschaftstheorie. Dagegen kann wiederum die interpersonelle Produktionswelt angeführt werden. Ein Vergleich ist hier alleine dadurch nicht möglich, dass kein eigentliches Angebot der hier produzierenden Firmen besteht. Denn das Angebot ergibt sich eigentlich erst mit dem Beginn der Transaktion, bei welcher anhand der Eigenheiten des Käufers das Produkt definiert wird. Dadurch ist keine rationale Wahl im Sinne der industriellen Produktionswelt möglich. Rational ist es hier eher, auf bewährte Produzenten zu setzen, welche die spezifische Situation des Käufers gut kennen und so ein hoch spezialisiertes Produkt - welches die eigentliche Qualität in dieser Welt darstellt - herstellen können. Die Marktwelt nimmt hierbei eine Mittelposition ein. Die Produktion findet hier ebenfalls spezialisiert statt, jedoch besteht ein an den Kundenbedürfnissen ausgerichtetes Marktangebot. Das Angebot hat folglich nicht die generische Reichweite der industriellen Produktionswelt, da es an den schnell schwankenden Bedürfnissen der Konsumenten ausgerichtet ist, aber es besteht dennoch im Vergleich zur interpersonellen Welt ein sichtbares, wenn auch begrenzt vergleichbares Angebot.

Auch für die Produzenten besteht ein unterschiedliches Maß an Unsicherheit. Storper und Salais beziehen sich in diesem Punkt auf die Trennung von „Risiko“ und „Unsicherheit“ von Knight. „Risiko“ bezeichnet hier eine quantifizierbare Unsicherheit, während „Unsicherheit" eine unmessbare Unsicherheit bezeichnet (Knight 2006, S. 19 f.). Generische Welten sind für Storper und Salais durch Risiko, während gewidmete Welt durch Unsicherheit gekennzeichnet sind (Storper und Salais 1997, S. 27 ff.). Dies wird am Unterschied zwischen der Marktwelt und der industriellen Welt deutlich. Da sich hier die Produktion an allgemeinen Standards ausrichtet, stellt sich lediglich die Frage nach der Nachfragehöhe. Im Gegensatz dazu stellt sich bei der Marktwelt generell die Frage, ob eine Nachfrage nach den produzierten Produkten in Zukunft überhaupt noch besteht durch die sich ständig ändernden Konsumentenwünsche. Es ist hier folglich durchaus denkbar, dass die Nachfrage nach einem Produkt komplett wegfällt. 
Storper und Salais führen zudem unterschiedliche Konventionen der Arbeit in Produktionswelten ein. $\mathrm{Zu}$ beachten ist dabei die bereits dargestellte breitere Verwendung des Konventionenbegriffs im Vergleich zum Konzept der Rechtfertigungsordnung (vgl. hierzu Abschn. 3.1.1). Die unterschiedlichen Konventionen der Arbeit in den verschiedenen Produktionswelten orientieren sich an den grundlegenden Strukturierungsprinzipien der jeweiligen Welt. Arbeitsabläufe in der industriellen Welt sind stark strukturiert und standardisiert. Dagegen spielen beispielsweise die Herkunftsregion der Mitarbeitenden oder persönliche Beziehungen keine Rolle. Arbeiter verstehen sich als Angestellte an Arbeitsstationen. Charakteristisch für die Marktwelt sind flexible Beschäftigungszeiten und Zeitarbeitsverträge, welche ein flexibles Eingehen von Unternehmen auf die aktuelle Marktlage ermöglicht. Die Arbeiter verstehen sich hier als verfügbare, autonome Individuen. Die interpersonelle Welt ist im Hinblick auf die Arbeit weniger stark standardisiert und strukturiert als etwa die industrielle Welt. Die Integration der Mitarbeitenden geschieht über eine geteilte Wertbasis und durch die den Mitarbeitenden eigene Expertise werden Arbeitsschritte oft durch eine Person zusammenhängend ausgeführt. Mitarbeiter sind Mitglieder einer Arbeitsgemeinschaft. In der Welt der intellektuellen Ressourcen beispielsweise wird der Lohn für die Arbeit nicht anhand des Outputs festgelegt, sondern ist grundsätzlich eine Investition in die Zukunft des Unternehmens. Arbeiter verstehen sich in dieser Welt als Experten (Storper und Salais 1997, S. 59 ff.).

Innovation findet in allen Produktionswelten statt. Es ist also nicht etwa nur die Welt der intellektuellen Ressourcen, welche Innovationen hervorbringt. Vielmehr muss Innovation innerhalb der Prinzipien einer Welt verstanden werden. Innovation ist folglich nur durch den Bezug auf die vorherrschenden Rechtfertigungsordnungen verstehbar und beschreibt die relational bessere Verwirklichung der jeweiligen Prinzipien innerhalb einer Produktionswelt (Storper 1996, S. 771). Innovation bedeutet dadurch einen Wissensvorsprung in einer Produktionswelt.

Generell kann hier festgehalten werden, dass Produktionswelten eigene Entwicklungspfade verfolgen. Dies betrifft beispielsweise die Reaktion auf staatliche Vorgaben und Steuerungsbemühungen. Bailly et al. konnten aufzeigen, inwiefern sich die einander scheinbar sehr ähnlichen Arbeiten der an Privathaushalten ausgerichteten Haushaltsarbeiten und der ebenfalls an Privathaushalten ausgerichteten Care-Dienstleistung grundlegend unterscheiden im Hinblick auf Lohnperspektive. ${ }^{57}$ Bailly et al. führt dies auf die unterschiedliche produktionsweltliche Einbettung

\footnotetext{
${ }^{57}$ Deutlich wird damit auch, dass sich das Erklärungspotential der Produktionsweltentheorie auch auf Dienstleistungen übertragen lässt. Vgl. hierzu Gadrey (1996).
} 
zurück. Die Haushaltsarbeiten verorten sie in der Marktwelt, während dem die Care-Dienstleistung in der interpersonalen Welt verortet werden. Dies führt dazu, dass die Care-Dienstleistung weniger standardisiert werden können, da sich deren Qualität erst in der Arbeit selbst zeigt. Dadurch ist es auch schwieriger, in dieser Welt arbeitende Personen zu ersetzen. Denn dass die persönliche Beziehung zwischen Pflegenden und Gepflegten funktioniert und insbesondere für die Gepflegten angenehm ist, lässt sich nicht bereits vor dem Arbeitsantritt beurteilen. Entsprechend ist der Wechsel von Pflegepersonal stets mit einem Risiko verbunden. Die Putzdienstleistungen dagegen sind standardisiert, in ihrem Erfolg einfacher überprüfbar und die dort tätigen Arbeitenden einfacher austauschbar, was gemäß Bailly et al. zum niedrigen und prekären Lohnniveau führt und gescheiterten Versuchen des Staates, diese Situation zu ändern (Bailly et al. 2013). Insgesamt wird durch die Studie von Bailly et al. deutlich, dass Produktionswelten auf Regulierungen und Steuerungsbemühungen unterschiedlich reagieren und in verschiedenen institutionellen Settings auch unterschiedlich erfolgreich sein können.

Robert Salais und Dorothée Rivaud-Danset beschrieben für die verschiedenen Produktionswelten auch unterschiedliche Finanzierungskonventionen. Salais und Rivaud-Danset sehen für die Unternehmen drei Möglichkeiten, liquide zu bleiben. Dies ist erstens möglich durch die Bildung von Rücklagen innerhalb des Unternehmens, es ist zweitens möglich durch die Finanzierung am Finanzmarkt oder durch Risikokapitalgeber und schlussendlich durch die Finanzierung einer Bank. Innerhalb der Banken unterscheiden Salais und Rivaud-Danset zwei unterschiedliche Idealtypen: Die engagierte und die allein nach Aktenlage verfahrende Bank. Bei jeder der Produktionswelten identifizieren sie eine Finanzierungskonvention und unterscheiden zwischen der industriellen Konvention des Finanzmarktes, der vertraglichen Finanzierungskonvention und der Konvention der interpersonellen Finanzierung. So finanziert sich beispielsweise die interpersonelle Produktionswelt auf Basis der Konvention der engagierten Bank. Denn anders als die nach Aktenlage verfahrende Bank, beurteilt die engagierte Bank nicht nur aufgrund von ,harten Zahlen“, sondern steht dem zu finanzierenden Unternehmen als Ratgeber zur Seite. Zugleich kennt man sich gegenseitig vertieft durch eine jahrelange Zusammenarbeit, was einen Vertrauensvorschuss bewirkt (Rivaud-Danset und Salais 1992). ${ }^{58}$ Am Beispiel dieser Finanzierungskonventionen wird der eigentliche Weltencharakter von Produktionswelten anschaulich: Die Konventionen der Produktionswelten zeigen sich nicht nur in der direkten Transaktionsbeziehung zwischen Käufer und Verkäufer, sondern

\footnotetext{
${ }^{58}$ Vgl. hierzu für eine deutsche Zusammenfassung Diaz-Bone (2018, S. 230 ff.).
} 
begründen vielmehr eine eigene Wirtschaftssphäre, welche Zulieferer, Banken, lokale Wirtschaftsförderung etc. miteinschließt und so insgesamt ein transorganisationales Wirtschaftsnetzwerk fundiert.

Francois Horn überträgt das Produktionsweltenmodell von Storper und Salais auf die Produktion von Software. Er unterscheidet vier unterschiedliche Welten: Die interpersonelle Welt, die fordistische Welt, die Welt der Entwicklung (franz. „création“) und die Welt der flexiblen Produktion (Horn 2001, S. 45 ff.). Zentral ist dabei die Feststellung, dass sich die Entwicklungswelt oft außerhalb des eigentlichen „Marktes“59 befindet, das heißt insbesondere an Universitäten und weiteren staatlich finanzierten Forschungsstellen oder auf der Basis von Open-Source-Projekten existiert (Horn 2001, S. 49, 2004, S. 18). Denn hier werden Grundlagenentwicklungen durchgeführt, welche kaum durch kommerziell orientierte Organisationen finanziell und risikotechnisch getragen werden könnten. Zudem dynamisiert er das Produktionsweltenmodell von Storper und Salais und verweist auf verschiedene Entwicklungen von Softwareprodukten, welche dazu führen können, dass Produkte und dahinterstehende Organisationen eine produktionsweltliche Transformation durchlaufen. ${ }^{60}$ Infolge einer Industrialisierung werden so beispielsweise Produkte der interpersonellen Welt zu Produkten der fordistischen Welt transformiert. Eine Transformation findet beispielsweise auch dann statt, wenn infolge einer Vermarktlichung, bzw. einer Verwertung (franz.: „Valeurisation“), eine Aufbereitung der Entwicklungen der Entwicklungswelt für einen kommerziellen Markt stattfindet und so eine Transformation von der Entwicklungswelt hin zur Welt der flexiblen Produktion stattfindet (Horn 2001, S. 52).

Deutlich wird mit den Arbeiten von Horn, dass sich Akteure und Organisationen, aber auch einzelne Projekte nicht statisch auf eine gewisse Produktionswelt beziehen. Vielmehr finden auch Transformationen statt, welche jedoch koordinative Anpassungen notwendig machen. ${ }^{61}$ Werden Produktionswelten auf weitere Konzepte der EC bezogen, so wird deutlich, dass Produktionswelten sowohl Rechtfertigungsordnungen wie auch Regime des Engagements in spezifischer Weise integrieren müssen. Zudem wird deutlich, dass Forminvestitionen

\footnotetext{
${ }^{59} \mathrm{Im}$ basalen Sinne des Verkaufs von Produkten.

${ }^{60}$ Jonathan Murdoch und Mara Miele nehmen ebenfalls eine dynamische Produktionsweltenperspektive ein und zeigen anhand zweier Fälle unterschiedliche produktionsweltliche Transformationen im italienischen Lebensmittelmarkt nach (1999).

${ }^{61}$ Solche Transformationen im Hinblick auf Survey-Welten werden in Abschn. 7.1 dargestellt.
} 
(Thévenot 1984) ein unterschiedlich starkes Gewicht, bzw. eine unterschiedliche Bedeutung in den verschiedenen Produktionswelten zukommt. Die industrielle Welt basiert maßgeblich auf Standards (welche als Forminvestitionen verstanden werden können) mit einer hohen soziogeographischen Reichweite, während Forminvestitionen in der interpersonellen Welt eine viel geringere Reichweite aufweisen.

\subsubsection{Tatsächliche Produktionswelten und Organisationen}

Storper und Salais unterscheiden zwischen möglichen und realen Produktionswelten. Mögliche Produktionswelten stellen die vier im letzten Kapitel dargestellten Produktionswelten dar. Mögliche Produktionswelten können als Idealtypen einer Produktionswelt verstanden werden, welche kaum real anzutreffen sind. Am Beispiel der Design- und Handwerksindustrie in Norditalien, der Fashion- und High-Tech Industrie in Paris und der High-Tech Industrie im Silicon Valley analysieren Storper und Salais fallbezogen reale Produktionswelten (1997, S. 97 ff.). ${ }^{62}$ Reale Produktionswelten - bzw. in einem zweiten Schritt Organisationen - sind gerade dadurch gekennzeichnet, dass sie mögliche Produktionswelten kombinieren und so Kompromisse zwischen ihnen eingehen (Storper und Salais 1997, S. 77 ff.). Diese Kompromisse müssen durch Organisationen etabliert und auf Dauer gestellt und gegebenenfalls dem Markt angepasst werden. Der Kompromisscharakter von realen Produktionswelten stammt jedoch nicht zuletzt daher, dass Organisationen typischerweise in ein weitläufiges Produktionsnetzwerk eingebunden sind mit verschiedenen Zuliefern und Abnehmern. Diese können auf der Basis von anderen Produktionswelten produzieren. Daher ist eine Übersetzungsfähigkeit der verschiedenen Organisationen notwendig, d. h. einerseits die Kenntnisse der verschiedenen Produktionskonventionen, andererseits die Fähigkeit, die eigenen Anforderungen in die Logik der konventionell unterschiedlich angeleiteten Produktionsart übersetzen zu können (Salais und Storper 1992). Es ist diese Kompromisserstellung und -bewältigung, welche der Organisation in der EC eine Zwitterstellung verleiht. Organisationen aus der Perspektive der Produktionsweltentheorie wie auch der EC im Allgemeinen nicht die

\footnotetext{
${ }^{62}$ Die Produktionsweltentheorie war außerdem Grundlage für Studien zu Agrarmärkten (Trabalzi 2007), zu Theaterwelten (Urrutiaguer 2014) zu Software-Welten (Horn 2004) und zu Rechtswelten (Didry 2002).
} 
grundlegende Untersuchungsebene (Diaz-Bone 2018, S. 185 ff.; Storper und Salais 1997, S. 44). ${ }^{63}$ Sie wird nicht als ontologische Gegebenheit verstanden und von Märkten unterschieden. ${ }^{64}$ Organisationen sind aus der Perspektive der EC keine einheitlichen Akteure, sondern ihnen kommt vielmehr die Aufgabe der Integration von verschiedenen Rechtfertigungsordnungen, bzw. Produktionswelten zu (Diaz-Bone 2018, S. 185 ff.; Thévenot 2001). Andererseits kommt der Analyse von Organisationen gerade durch die Konfliktbewältigung und der Kompromisserstellung zwischen Konventionen ein hoher Stellenwert zu, da hier (gesamt-) gesellschaftliche Konflikte bewältigt werden.

Organisationen werden in der EC als „,compromising device“ konzipiert (Thévenot 2001, S. 410). Damit wird die zwischen verschiedenen Konventionen vermittelnde Funktion von Organisationen beschrieben, welche Organisationen übernehmen. Ein zentraler Kompromiss in produktionsorientierten Organisationen ist beispielsweise die Vermittlung der marktweltlichen Konvention an die industrielle Konvention. Da sich in der marktweltlichen Konvention der Wert von Gütern sehr schnell ändern kann, ist eine Vermittlung an die industrielle Rechtfertigungsordnung notwendig. Denn die industrielle Rechtfertigungsordnung ist an zeitlich länger dauernden Produktionszyklen orientiert, um die Nachfrage in konstant gleichbleibender Qualität und in der benötigten Anzahl liefern zu können (Eymard-Duvernay 2004). Organisationen kommt so eine wichtige Koordinationsfunktion in der Gesellschaftstheorie der EC zu, da hier Kompromisse auf Dauer gestellt werden und Organisationen so als Dispositive für gesellschaftliche Vorgänge dienen.

Die Pluralität der durch eine Organisation integrierten Welten oder Konventionen macht für die in dieser Organisation beschäftigten Personen einen Sinn für diese Pluralität notwendig. Claude Didry spricht im Hinblick auf die von ihm identifizierten Welten von einer „legalen Sensibilität“ (Didry 2002, S. 76, 91). Denn die basale Voraussetzung für das Etablieren von Kompromissen besteht in der Kenntnis der verschiedenen zu integrierenden Logiken. Die Integration von verschiedenen produktionsweltlichen Logiken in tatsächlichen Produktionswelten hat dadurch zur Folge, dass die in einer Organisation tätigen Personen auch einen praktischen Sinn haben können (oder müssen) für Produktionswelten, welche nicht schwergewichtig von der entsprechenden Organisation bedient wird.

\footnotetext{
${ }^{63}$ D. h. sowohl der Produktionsweltenperspektive wie der Rechtfertigungsperspektive. Einen umfassenden Überblick zur EC informierten Organisationsforschung liefern Søren Jagd (2011) und Knoll (2015b).

${ }^{64}$ Darin besteht eine wichtige Differenz zum Neoinstitutionalismus (Knoll 2015a, S. 18 ff.).
} 
Zentral für die Analyse von Organisationen ist das Konzept der Forminvestition. Denn mit der Investition werden Konventionen dauerhaft aneinander vermittelt und auf Dauer gestellt. Im Hinblick auf Märkte ergibt sich eine dreistufige Formenanalyse. Zunächst sind Formen zentral für die Organisation der Produktion, indem hier Vorgänge und Prozesse definiert werden (Thévenot 2002, S. 9). Forminvestitionen stellen demgemäß eine kognitive Instrumentierung für die Mitarbeitenden einer Organisation dar mit dem Ziel einer kollektiven Unsicherheitsbewältigung (Eymard-Duvernay 2004). Formen sind zudem zentral für das Verständnis der interorganisationalen Koordination. In fehlenden oder unvollständigen Formen können Koordinationsprobleme zwischen Organisationen lokalisiert werden. Zudem lassen sich auch Koordinationsprobleme zwischen Firmen auf unterschiedliche Formen zurückführen (Thévenot 1984, S. 14). Drittens sind Märkte auf dauerhafte und interpretationsresistente Formen angewiesen (Thévenot 2002, S. 9). Wie die Produktionsweltentheorie aufgezeigt hat, ist dies jedoch stark von der jeweiligen Produktionswelt abhängig. Formen mit hoher Abstützung sind in der industriellen Welt zu finden, während in der interpersonellen Welt keine Marktformen vorhanden sind.

\subsection{Wertigkeitsproduktion}

[...] il n'y a pas, en toute généralité, de coordination des comportements sans coordination des jugements sur les comportements [...] (Bessy und Favereau 2003, S. 131).

Ein zentrales Thema der EC ist die Kritik an der neoklassischen Konzeption von Qualität. Kritisiert wird hierbei die Annahme einer objektiv feststellbaren Qualität von Produkten (Eymard-Duvernay 1989). Damit einher geht die Proklamation einer Ökonomie der Qualitäten, welche radikal unterschiedliche Produktqualitäten beschreibt. Diese Qualitätsperspektive der EC wird im folgenden Unterkapitel dargestellt. Hier werden folglich die Grundlagen geschaffen für das in Abschn. 2.5.5 eingeführte dritte Element einer pragmatischen Konzeption von Survey-Qualität, dem Wissensformat, bzw. den Konventionen des Wissens. Im Gegensatz zur Perspektive der klassischen Survey-Methodologie auf Survey-Qualität wird dabei deutlich, dass die EC stets von einer radikalen Pluralität an Qualitätsvorstellungen ausgeht. Auch die Qualität von Surveys lässt sich folglich erst durch den Bezug auf eine Konvention (des Wissens) evaluieren. Diese durch die EC eingenommene Perspektive auf die Qualität von Produkten wird durch den Einbezug von Konzepten der Global-Value-Chain- 
Forschung (kurz: GVC) durch eine dezidierte Produktionsperspektive ergänzt. Analysiert werden hier Produktionsabläufe im Hinblick auf ihre Fundierung durch Rechtfertigungsordnungen sowie auch die Transformation dieser Fundierung während des Produktionsprozesses. Auch Surveys werden auf der Basis einer zunehmend komplexen und auf viele Stationen distribuierten Produktionsweise hergestellt (Desrosières 2000, S. 183). Ein weiteres wichtiges Konzept der GVC stellt dasjenige der Governance dar. Mit Blick auf die unterschiedlichen Koordinationsprozesse wird damit deutlich, dass die Governance in den verschiedenen Survey-Welten auf unterschiedliche Art und Weise organisiert ist und insbesondere auch unterschiedlich stark durch die Auftraggeber kontrolliert wird. Der Miteinbezug von Konzepten der GVC-Forschung liefert dadurch ein tieferes Verständnis der Produktionskette von Surveys. Im letzten Unterkapitel wird die Theorie der Distributed Cognition vorgestellt. Denn trotz, bzw. gerade wegen der Pluralität an existierenden Survey-Welten ist die Herstellung einer einheitlichen kollektiven Orientierung an survey-weltlichen Konventionen zentral für das Gelingen von Survey-Projekten. Die Theorie der Distributed Cognition stellt für das Herstellen einer auf verschiedene Akteure verteilten kollektiven Orientierung eine Detailperspektive bereit.

\subsubsection{Die Produktion von "Qualität“}

Die EC begründete eine Theorie des Wettbewerbs um Qualitäten. ${ }^{65}$ Denn während die neoklassische Wirtschaftstheorie von einem totalen Wettbewerb in einem Markt ausgeht, verweist die EC auf nicht substituierbare Produkte. Während die These der nicht-substituierbaren Produkte mit Blick auf Produktionswelten zunächst kaum evident erscheint, da es sich bei den behandelten Produkten (High-Tech, Lederwaren und Haute-Couture Kleidung) offensichtlich nicht um substituierbare Produkte handelte, zeigte an die Theorie der Produktionswelten und der Rechtfertigungsordnungen anschließende Forschung die eigentliche Qualitätsblindheit neoklassischer ökonomischer Forschung auf (Diaz-Bone 2018, S. 148). Die grundlegende Position der EC ist hier, dass Produkte und Dienstleistungen erst durch den Bezug auf eine Rechtfertigungsordnung, bzw. eine Konvention, bewertet werden können. Pionierarbeit leisteten

\footnotetext{
${ }^{65} \mathrm{Vgl}$. auch die Markttheorie von Harrison White und daran anschließende Vergleiche und Diskussionen der EC (Biencourt et al. 2002; Diaz-Bone 2010d; Godart und White Harrison C. 2007).
} 
hierbei Pierre Boisard und Marie-Thérèse Letablier (Boisard und Letablier 1987). Sie zeigten in empirischer Forschung zwei unterschiedliche Produktionslogiken des Camemberts de Normandie auf. Während bei der einen Produktionsart Effizienz, Kontrolle und Lebensmittelsicherheit im Vordergrund stehen, ist es bei der zweiten Herstellungsart stärker die Orientierung an einer ursprünglichen Art der Herstellung und am Konzept des Terroirs. ${ }^{66}$ Boisard konnte jedoch aufzeigen, dass es sich bei den verschiedenen Produktionsarten nicht lediglich um die Differenz zwischen effizienten gegenüber weniger effizienten Produktionsarten handelt, sondern um grundlegend verschiedene Produktionsphilosophien. Die erste Herstellungsart basiert maßgeblich auf der industriellen Rechtfertigungsordnung, während die zweite maßgeblich auf der häuslichen Rechtfertigungsordnung beruht. Durch die, ,ineffiziente“67 Produktion der traditionellen Herstellungsart - welche sich zudem üblicherweise in kleineren Käsereien abspielte - drohte diese unterzugehen. Doch es war gar nicht das Ziel der kleineren, traditionellen Käsereien, effizient und industriell zu produzieren. Es war vielmehr das Ziel, authentischen und handwerklich hervorragend produzierten Käse herzustellen, bei dem man die Herkunft herausschmeckt. Die handwerklichen Produkte gerieten so in eine Konkurrenz zu den industriell hergestellten Produkten, ohne dass die eigene spezifische Qualität sicht- und erlebbar würde. Interessant ist, dass die industriellen Produzenten ein Interesse daran haben, dass der traditionelle Camembert weiterhin produziert wird. Denn symbolisch werben auch die industriellen Produzenten mit dem traditionellen Bild des Camemberts, was als Werttransfer bezeichnet werden kann. Der Kernpunkt der Unterscheidung zwischen der industriellen und der handwerklichen Produktionsweise stellt die Pasteurisierung dar. Aus Sicht der industriellen Hersteller ist die Pasteurisierung ein Mittel, um die Lebensmittelsicherheit ${ }^{68}$ und die industrielle Verarbeitung durch das Ausfällen der Proteine zu gewährleisten. Aus Sicht der handwerklichen Hersteller werden mit der Pasteurisierung jedoch die Eigenheiten der Milch zerstört, da dadurch die unterschiedlichen Herkunftscharakteristika eingeebnet werden. Sie verunmöglicht zudem auf

\footnotetext{
${ }^{66}$ Vgl. zum Konzept des Terroirs Barham (2003, S. 131 ff.).

${ }^{67}$ Was als Kritik der industriellen Konvention an der handwerklichen Konvention gelesen werden kann.

${ }^{68}$ Boisard zitiert einen stolzen Hersteller von industriellen Camembert: „Here no one ever touches our product“ (1991, S. 180). Im Gegensatz dazu ist es bei der Handwerklichen Herstellung gerade auch die haptische Beurteilung des Käsers, welche zur Qualität des Endproduktes beiträgt (ebd., S. 184).
} 
technischer Ebene die traditionelle Herstellungsart (Boisard 1991, S. 178). Dem Verschwinden der kleineren Käsereien liegt demzufolge nicht zwingend ein fehlender Modernisierungsschub zu Grunde. Wie sich herausstellen sollte, war es die fehlende Kommunikation dieser spezifischen Herstellungsweise. Durch die fehlende Kommunikation ging der Käse im großen Angebot der industriell hergestellten Camemberts unter, was auch durch den viel höheren Preis begründet ist. ${ }^{69}$ Die Einführung des AOC-Labels versuchte dann, diesen Missstand zu beheben. In der Folge stieg auch der Absatz rapide an. Boisard zweifelt jedoch daran, dass dies auf das AOC-Label zurückzuführen ist (Boisard 2003, S. 185 f.).

\subsubsection{Global-Quality-Chains}

Der analytische Mehrwert der Produktionsweltentheorie liegt im Aufzeigen von systematisch unterschiedlichen Produktionsprozessen und damit einhergehenden unterschiedlichen Produktqualitäten. Problematisch für die vorliegende Forschung ist jedoch eine starke Produktionsperspektive, in welcher die schlussendlich beim Kunden angekommene, bzw. die durch ihn evaluierte Produktqualität kaum thematisiert wird (Ponte und Gibbon 2005). So wird in der Theorie der Produktionswelten nicht darauf eingegangen, wie beispielsweise die stark gemäß den Konventionen der interpersonellen Welt produzierten Güter der norditalienischen Lederindustrie im Verkauf (noch) der handwerklichen Konvention entsprechen. Die von den Kunden wahrgenommene Produktqualität wird folglich nicht ausreichend thematisiert. Die Theorie der Produktionswelten wird aus diesem Grund durch die Global-Value-Chain-Perspektive ergänzt. ${ }^{70}$ Diese bringt einen Fokus auf verschiedene Stationen des Produktionsprozesses bis hin zur schlussendlich durch den Konsumenten erlebten Produktqualität ein und ermöglicht so die Analyse der Abfolge von unterschiedlichen Qualitätsregimen während des gesamten Produktionsprozesses.

Die Global-Value-Chain-Forschung beschäftigt sich mit der Organisation von (globalen) Transaktionsprozessen. Dabei ist es das Ziel, sämtliche Tätigkeiten (wie beispielsweise Design, Produktion, Marketing oder auch Support der

\footnotetext{
${ }^{69}$ Boisard spricht von einem ungefähr dreifach so hohen Preis für traditionell hergestellte Camemberts im Vergleich zu industriell hergestellten (1991, S. 188).

${ }^{70}$ Insgesamt stellt die Theorie der Global-Quality-Chains einen idealen konzeptuellen Rahmen für die Analyse von statistischen Ketten dar, wie dies in den beiden Fallanalysen angestrebt wird. Dieses Konzept wurde durch Thévenot eingeführt im Hinblick auf die distribuierte Produktion von statistischen Daten (1983).
} 
Endverbraucher) von Firmen zu analysieren, welche zum schlussendlichen Produkt beitragen (Gereffi und Fernandez-Stark 2011). Im Gegensatz zu Coase und Williamson und im Anschluss an die Commodity-Chain-Forschung beschreibt sie verschiedene Netzwerkformen, welche zwischen den klassischen Organisationsformen der Hierarchie - also der integrierten Organisation - und des Marktes lokalisiert werden können (Sturgeon 2009, S. 122). Die verschiedenen Governance-Modelle von Value-Chains werden auf der Basis dreier Dimensionen analytisch herausgearbeitet: Komplexität der Transaktion, Möglichkeit der Kodifizierbarkeit von Transaktionen und den Fähigkeiten der Versorgungsbasis. Anhand dieser Dimensionen werden fünf Governance-Formen von Firmenrelationen beschrieben: Markt, Modular, Relational, Captive und Hierarchy (Gereffi et al. 2005, S. 87).

Das Global-Value-Chain Modell wurde aus der Perspektive der EC im Hinblick auf die Annahme einer objektiven Produktontologie kritisiert. Aus der Perspektive der EC ergibt sich eine Produktontologie erst durch den Bezug auf eine Konvention und liegt also nicht im Produkt selber vor. Die verschiedenen Dimensionen von Global-Value-Chains sind damit nicht unabhängig von der konventionellen Basis zu sehen (Diaz-Bone 2013, S. 438). Im Anschluss an die Kritik der EC an der Global-Value-Chain-Forschung wurde in verschiedenen Publikationen eine EC-Perspektive auf Value-Chains entworfen, welche als Quality-Convention-Chain benannt werden kann (Diaz-Bone 2013, S. 441). Zentral sind aus Sicht der EC bei der Analyse von Value-Chains Regeln und Bedingungen der Teilnahme der verschiedenen involvierten Akteure (Ponte und Gibbon 2005, S. 3). Dabei werden sämtliche die Value-Chain mitstrukturierenden Entitäten miteinbezogen. So verweist Ponte auf die mitstrukturierende Rolle des bekannten Weinkritikers Robert Parker für die Value-Chains von Weinen, insbesondere solchen der gehobenen Preiskategorie, obwohl Parker lediglich seine Meinung und Bewertung einbringt (Ponte 2009, S. 247). Dabei lassen sich Unterschiede zwischen verschiedenen Value-Chains, aber grundsätzlich auch zwischen verschiedenen Abschnitten einer Value-Chain untersuchen (Ponte und Gibbon 2005, S. 3). Eine EC-basierte Untersuchung von Value-Chains besteht folglich in der Identifikation der grundlegenden Rechtfertigungsordnung einer Value-Chain und deren Einfluss auf die GovernanceFormen einer Value-Chain (Ponte 2009). ${ }^{71}$

\footnotetext{
${ }^{71}$ So wurde mit diesem Ansatz beispielsweise dargestellt, warum trotz erhöhter Nachfrage nach Kaffee die Erlöse für die Produzenten sanken (Daviron und Ponte 2005). Vgl. für einen Überblick zur EC-Perspektive auf Global-Value-Chains Diaz-Bone (2018, S. 203 ff.).
} 
Eine zentrale Dimension bei der Analyse von Value-Chains stellt die Reichweite von Rechtfertigungsordnungen dar. So unterscheiden sich beispielsweise die häusliche und die industrielle Welt stark im Hinblick auf ihre Reichweite, das heißt die weiterführende Kommunizierbarkeit (Ponte 2009, S. 252 ff.). Die industrielle Welt hat durch allgemein bekannte Standards eine sehr hohe Reichweite. Die hier produzierte Qualität ist dadurch weit und über verschiedene Stationen einer Value-Chain hinweg kommunizierbar, was insbesondere der einfachen Quantifizierbarkeit dieser Welt geschuldet ist (Boltanski und Thévenot 2007, S. 276 ff.). Dagegen ist der Transport der spezifischen Qualität der häuslichen Welt nur sehr bedingt möglich (Boltanski und Thévenot 2007, S. 229 ff.). Bedingt durch den Qualitätsursprung in spezifischen lokalen Traditionen ist ein Transport der Qualität ohne deren Kenntnis nicht möglich. Dies ist der Grund, warum hier oft in Form von Labels ein Kompromiss mit der industriellen Rechtfertigungsordnung eingegangen wird.

Auf der Basis des Global-Value-Chain-Ansatzes kritisieren Stefano Ponte und Peter Gibbon das Produktionsverständnis der EC. Gemäß ihnen ist die Produkt- von der Produktionsqualität zu trennen (Ponte und Gibbon 2005, S. 16). Value-Chains sind demzufolge oft gerade durch eine Abfolge von verschiedenen Rechtfertigungsordnungen gekennzeichnet. Dadurch ist es durchaus wahrscheinlich, dass unterschiedliche Rechtfertigungsordnungen den Produktions- und den Verkaufsprozess organisieren. Diese Eigenschaft von Value-Chains wurde beispielsweise bei Kaffee nachgewiesen (Daviron und Ponte 2005, S. 219 ff.; DiazBone 2013, S. 442).

\subsubsection{Distributed Cognition und Workplace Studies}

Zu Beginn des Kapitels zu den Global-Quality-Chains wurde auf deren Ergänzung der Produktionsweltenperspektive durch den Fokus auf den über verschiedenen Stationen ablaufenden Produktionsprozess verwiesen. Die Quality-Chain-Analyse ermöglicht so eine Analyse verschiedener Qualitätsregime während des Produktionsprozesses. Damit ermöglicht die Quality-Chain-Analyse zwar eine diachrone Perspektive auf das Prozessieren verschiedener Konventionen während des Produktionsprozesses, ihr fehlt jedoch eine en-detail-Perspektive auf die Mobilisierung von Konventionen, Akteuren, Formen und Objekten in den verschiedenen Stationen des Produktionsprozesses. Aus diesem Grund wird die Produktionswelten- und Quality-Chain-Perspektive durch die Theorie der Distributed Cognition und Workplace Studies ergänzt. Diese beiden verwandten Perspektiven (Heath und Luff 2000, S. 8) bringen eine Detailperspektive auf die 
Herstellung von Survey-Qualität und die Mobilisierung von survey-weltlichen Entitäten wie Objekten, Formen und Konventionen ein. Obwohl beide Perspektiven starke Überschneidungen aufweisen, lassen sich dennoch Unterschiede feststellen. So fokussiert die Distributed Cognition stark auf die über verschiedene Situationen distribuierte Koordination von Prozessen, während sich die Workplace Studies stärker auf die Hervorbringung von Ordnung in Arbeitsprozessen in einzelnen Situationen fokussiert. Die folgenden Ausführungen dienen einer Mikrofundierung des Situationalismus der EC. Denn sowohl die EC, die Distributed Cognition wie auch die Workplace Studies teilen eine situationsbasierte Erklärungslogik (Diaz-Bone 2011b; Hutchins 2000; Knoblauch und Heath 1999). Dadurch kann die EC von den Ausarbeitungen der Distributed Cognition und der Workplace Studies zur Koordination von transsituationalen Netzwerken und der verschiedenen Situationselemente profitieren. Zentral ist für die empirische Analyse dieser Arbeit die Identifikation der Distributed Cognition von objektgestützten Übersetzungsprozessen bei der transsituationalen Koordination sowie die Hervorhebung der Wichtigkeit der spezifischen Sozialisierung der verschiedenen in ein Koordinationsnetzwerk eingebundenen Akteure. Wichtige Beiträge für eine Mikrofundierung der Situationspragmatik der EC stellen zudem die aus der Ethnomethodologie entlehnten Konzepte der Reziprozität, der Reflexivität und der Unvollständigkeit von Regeln der Workplace Studies dar.

Kollektive kognitive Prozesse in Koordinationsnetzwerken werden von dem amerikanischen Psychologen Edwin Hutchins als „Distributed Cognition“ bezeichnet (Hutchins 1996). Hutchins stellte sich gegen eine Laborpsychologie, welche von der Kognition wie sie „In the wild“"72 stattfindet, abstrahiert. So untersuchte er beispielsweise die Navigation auf einem amerikanischen Kriegsschiff (Hutchins 1996) oder die Navigation in einem Flugzeugcockpit (Hutchins und Klausen 1998). Die grundlegende Idee von Hutchins bei der Untersuchung der Navigation auf einem amerikanischen Kriegsschiff ist, eine kollektive Kognition analog zu einer Individualkognition zu beschreiben. Im Unterschied zur Individualkognition sind bei der kollektiven Kognition die verschiedenen Schritte jedoch detailliert erfassbar, da sie in diesem Fall nicht verborgen innerhalb eines Akteurs stattfinden, sondern beobachtbar zwischen Akteuren erfolgt. Die kognitive Bewältigung der nautischen Navigation beschreibt Hutchins als Übersetzungen von Merkmalszuständen zwischen Medien und deren anschließende Gegenüberstellung (Hutchins 1996, S. 117 f.). Als Beispiel dient hier der Vorgang der Bedienung eines

\footnotetext{
${ }^{72}$ Wonach dann auch der Titel eines einflussreichen Buchs von Hutchins benannt wurde: „Cognition in the wild“ (Hutchins 1996).
} 
Sextanten. Dieser besteht aus drei Räumen: Dem geographischen Makroraum, dem Raum, welcher durch den Sextanten aufgespannt wird und dem Raum des Gyrokompasses (Hutchins 1996, S. 121 ff.). Die Funktion des Sextanten besteht darin, äußere Merkmale (geografische) in interne Merkmale (Grade) zu übersetzen. Die Grade können dann auf einer Karte eingetragen und mit Graden von anderen Navigationsinstrumenten oder einem zweiten Sextanten einander gegenübergestellt werden. Navigation kann so insgesamt als Übersetzungsleistung verstanden werden, in welcher verschiedene Zustände in eine Form gebracht werden, die es dem Kapitän erlaubt, Navigationsentscheidungen zu treffen. Maßgeblich dabei ist nebst der Verarbeitung von internen Navigationsdaten die Übersetzung von externen in interne Strukturen. Navigation lässt sich so als distribuierte Kognition bezeichnen und beschreiben, da auf dem Schiff eine Gruppe von Navigierenden in gemeinsamer Arbeit kognitiv auf die äußeren Umstände reagiert. Die Navigation - und damit die Kognition - ist folglich auf mehrere Personen und technische Artefakte verteilt und wird erst durch deren Zusammenarbeit möglich. In Anlehnung an Bruno Latour betont Hutchins die zentrale Rolle von technischen Artefakten bei der Schiffsnavigation. ${ }^{73}$ Es sind folglich die technischen Artefakte, welche den Großteil der Übersetzungsleistung bewältigen. Es sind dann auch die Seekarten, welche es ermöglichen, verschiedenste Informationen zusammenzutragen und zueinander in Beziehung zu setzen (Hutchins 1996, S. 111). Zudem lässt sich die distribuierte - wie auch die individuelle - Kognition nicht ohne eine kulturelle Einbettung und die daraus folgende Notwendigkeit einer An-Sozialisierung der Tätigkeit verstehen (Hutchins 1996, S. 111). Die Funktion des Sextanten ist folglich ohne die Kenntnis der (modernen) Standortangabe in Graden, Minuten und Sekunden nicht denkbar. Dies zeigt die enge Kopplung zwischen den technischen Instrumenten und der historisch entstandenen Kultur der Navigation.

Aus der Perspektive der Distributed Cognition wird deutlich, dass auch Survey-Projekte als eine distribuierte Kognition verstanden werden können. Denn die Survey-Koordination ist nicht nur auf verschiedene Personen verteilt, sondern oft auch auf verschiedene Organisationen. ${ }^{74}$ Die Perspektive der Distributed Cognition geht nun gerade davon aus, dass es zu einfach wäre, die Governance eines Survey-Projektes lediglich in der leitenden Person zu suchen, welche ,die Fäden zusammenhält". Vielmehr stützt sich die Governance von Survey-Projekten zunächst auf die spezifische Sozialisierung der beteiligten

\footnotetext{
${ }^{73}$ Hutchins verweist maßgeblich auf Science in action (Latour 1987).

${ }^{74}$ Dies wird besonders deutlich in Fall (B), in welchem sogar die Hotline für Befragte an eine andere Organisation ausgelagert wird (vgl. Abschn. 6.2.3).
} 
Personen - welche also zuerst in die spezifische Survey-Logik eingeführt werden mussten - und auf verschiedene Objekte ab. Im Hinblick auf den Bezug auf die Produktionsweltenperspektive wird jedoch deutlich, dass sowohl die Stützung der Governance, aber auch die Objektstützung, in den verschiedenen Survey-Welten, produktionsweltlich unterschiedlich ausgestaltet ist.

Eine neuere Perspektive zur Erforschung von Arbeit und Organisation stellen die Workplace Studies dar. Der zentrale Forschungsfokus ist hier die Untersuchung von kollektiven Arbeitsprozessen. Ein Fokus wird auf die Rolle moderner (Kommunikations-) Technik gelegt (Heath et al. 2000). Die Workplace Studies greifen elementar auf ethnomethodologische Konzepte zurück (Heath und Luff 2000; Rawls 2008). Dabei wird jedoch der starke Kommunikationsfokus der Konversationsanalyse korrigiert. Im Zentrum der Analyse stehen bei den Workplace Studies nicht alleine Kommunikationsprozesse, sondern die raumzeitliche und in eine materiale Umwelt eingebettete Hervorbringung von Arbeit. Untersucht werden folglich auch Kommunikationsprozesse, welche jedoch in ihrer zeitlichen und materialen Einbettung untersucht werden. Im Unterschied zur ethnografischen Forschung - welche ebenfalls die zeitliche und materiale Einbettung von Handeln betont - fokussieren die Workplace Studies jedoch auf die en-detail-Analyse von einzelnen Arbeitsabläufen. Zudem liegt das Ziel von Workplace Studies oft in einer direkten Verbesserung von Arbeitsabläufen (Harper 2000).

Eine wichtige Grundlegung der Workplace Studies stellt das Werk „Plans and situated actions" von Lucy Suchman (Suchman 2009) dar. Hier untersucht Suchman die Bedienung von Kopiergeräten. Suchman kritisierte die Perspektive auf die Bedienung von technischen Geräten, welche einen klaren Bedienungsplan als Eigenschaft von Akeuren voraussetzte. Sie zeigte demgegenüber auf ethnomethodologischer Grundlage auf, dass Akteure - analog zur menschlichen Kommunikation - stets eine situationsgestützte Interpretation der Geschehnisse vornehmen. Folgend ist es für die erfolgreiche Bedienung eines Kopiergerätes notwendig, eine konstante Rückmeldung zur aktuellen Situation, d. h. zur bereits getätigten Handlung und zur noch anstehenden Handlung zu erhalten. Handlung schließt folglich nicht nur die mentale Intention von Akteuren ein, sondern muss die objektgestützte Auseinandersetzung miteinbeziehen (Suchman 2009).

Die theoretische und methodologische Grundlegung der Workplace Studies hebt Anne Rawls hervor. ${ }^{75}$ Sie verweist in diesem Zusammenhang auf die

\footnotetext{
${ }^{75}$ Und sie kritisiert gleichzeitig die fehlende ausformulierte Theorieperspektive vieler Workplace Studies. In der Folge schlägt sie ein stärkeres Rekurrieren auf Harold Garfinkels Studies of Work und deren theoretischer Grundlegung durch die Ethnomethodologie vor (Rawls 2008).
} 
Ordnungsversessenheit (Begriff) der regulären Soziologie, welche Ordnung voraussetze, anstelle diese zu erklären (Rawls 2008). Sie stellt drei grundlegende ethnomethodologische Konzepte vor, welche für die Workplace Studies von Bedeutung sind. Zunächst geht sie auf den Herstellungsprozess von Kommunikation ein. Sie führt in diesem Zusammenhang das ethnomethodologische Konzept der Reziprozität ein (Rawls 2008, S. 712). Begriffe, Konzepte, Objekte, Sachverhalte etc. müssen demgemäß in der Kommunikationssituation zuerst hergestellt und referenziert werden durch deren grundlegende Indexikalität (Garfinkel 1984, S. 4 ff.). Erst danach sind sie für die intersubjektive Kommunikation verfügbar und es kann dann durch die Kommunikationsteilnehmer Bezug darauf genommen werden. Sie werden so zu „oriented objects“ (Garfinkel 1984, S. 713).

Ein weiteres zentrales von der Ethnomethodologie entlehntes Konzept der Workplace Studies ist gemäß Rawls das Konzept der Reflexivität. Dieses besagt, dass das Gesagte immer auf das bereits vorher Gesagte bezogen wird, d. h. seinen Sinn aus dem situativ hergestellten Kommunikationshorizont erhält (Rawls 2008, S. 719 ff.). Begriffe haben in diesem Verständnis folglich keine festen Bedeutungen, sondern deren Bedeutung wird in der Kommunikation selbst hergestellt. Zudem sind aus ethnomethodologischer Perspektive Regeln grundsätzlich unvollständig. Dies, da jegliche Situation notwendigerweise komplexer ist, als dass eine Regel je zu spezifizieren imstande wäre (Rawls 2008, S. 714). Ethnomethodologie - und dadurch auch die Workplace Studies - sind deswegen eine en-detail-Perspektive auf den Herstellungsaspekt, das „,doing“ von technikbasierter Kommunikation. Dabei gehen aus der Perspektive der Workplace Studies bei der Analyse von Arbeitsprozessen auch klassische Ethnographien zu wenig ins Detail (Rawls 2008, S. 725). Zentral ist eine sequenzielle Analyse, da sich der Kommunikationssinn aus dem Kommunikationsprozess selbst ergibt (Rawls 2008, S. 722).

Die Perspektive der Workplace Studies macht deutlich, dass die verschiedenen durch die EC beschriebenen Situationselemente situativ durch Akteure mobilisiert werden müssen. Zentral ist dabei, dass dieser Prozess interaktiv zwischen den verschiedenen involvierten Akteuren geschieht. Produktionsweltliche Objekte und Konventionen liegen demgemäß nicht „einfach“ in Situationen vor, sondern müssen durch die involvierten Personen aktiv mobilisiert werden.

\subsection{Spezifizierung des Forschungsinteresses}

In Kap. 2 wurde in unterschiedliche Perspektiven der Survey-Forschung eingeführt. Dabei wurde zwischen einer Datenperspektive, einer Prozessperspektive und einer survey-pragmatischen Perspektive auf Survey-Qualität unterschieden. 
Es wurde argumentiert, dass die von verschiedenen Survey-Praktikern beschriebene Kluft zwischen der akademischen Survey-Forschung und privatwirtschaftlich ausgerichteten Survey-Firmen eine survey-pragmatische Perspektive notwendig macht, um die unterschiedlichen Einbettungen und Logiken der Survey-Produktion identifizieren zu können. Abschn. 2.5.5 diente der konzeptionellen Grundlegung der Survey-Pragmatik. Das folgende Unterkapitel dient der Spezifizierung des Forschungsinteresses auf der Basis der in Kap. 3 aufgearbeiteten Theoriegrundlage. Dafür muss zuerst das Verhältnis der verschiedenen in diesem Kapitel aufgearbeiteten Theoriekonzepte geklärt werden.

Produktionswelten stellen das zentrale Konzept für die Untersuchung verschiedener Logiken der Survey-Produktion, d. h. die Organisation des Survey-Produktionsprozesses durch unterschiedliche Konventionen, dar. Verschiedene Produktionswelten haben eine unterschiedliche Organisation von Quality-Chains der Survey-Produktion zur Folge. Quality -Chains lassen sich wiederum nach einer unterschiedlichen Distributed Cognition unterscheiden, d. h. in einer unterschiedlichen Koordination von Konventionen, Objekten, Formen und Akteuren. Diese unterschiedliche Koordination der verschiedenen Situationselemente kann zugleich aus der Perspektive der verschiedenen Handlungsregime, bzw. Regime des Engagements, beschrieben und systematisiert werden. Der Unterschied zwischen den Konzepten Produktionswelten, Quality-Chains und Distributed Cognition liegt dabei im Grad der Abstraktion der Betrachtung der Koordination der verschiedenen genannten Situationselemente. Das Verhältnis der verschiedenen Konzepte wird in Abb. 3.1 systematisiert dargestellt.

In Abschn. 2.5.5 wurde argumentiert, dass gemäß einer survey-pragmatischen Perspektive Untersuchungskategorien nicht das Resultat einer (reinen) Orientierung am Untersuchungsgegenstand darstellen, sondern als Ergebnis

Abb. 3.1 Das Verhältnis zwischen den verwendeten theoretischen Konzepten

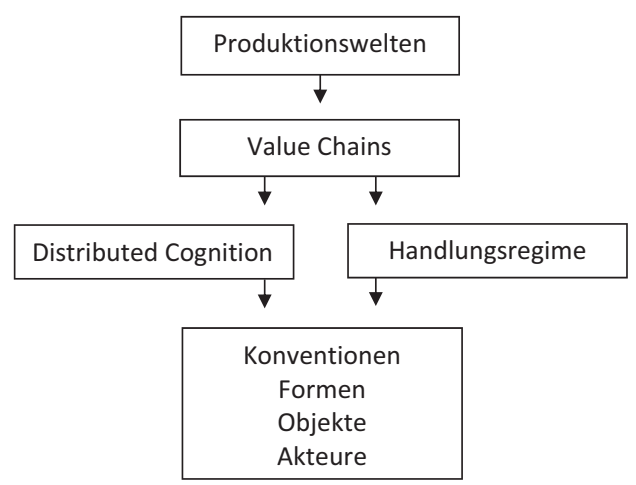


eines Ko-Konstruktionsprozesses betrachtet werden müssen. Diese Perspektive lässt sich durch die theoretische Grundlegung nun präzisieren. Aufbauend auf Desrosières Studien zu verschiedenen Statistik-Epochen (Desrosières 2009b) lässt sich festhalten, dass Erhebungskategorien durch eine Ko-Konstruktion zwischen Untersuchungsgegenstand, Forschungspraktiken und -instrumenten und der Forschung unterliegenden Konventionen erstellt werden. Anders als bei Desrosières stellt sich auf der Grundlage der Survey-Welten-Perspektive nicht nur die Frage nach einer Pluralität der Statistikkonventionen in temporaler Hinsicht, sondern auch in horizontaler Hinsicht. Auf der Basis einer Survey-Welten-Perspektive können unterschiedliche Koordinationsrahmen der Survey-Produktion angenommen werden. Es wird folglich keine Einheit der survey-basierten Statistikproduktion vorausgesetzt, sondern eine empirisch zu erfassende und beschreibende Pluralität der Wissensproduktion durch Surveys angenommen. Basierend darauf kann das erste, die empirische Forschung anleitende Forschungsinteresse formuliert werden:

Welche unterschiedlichen Survey- und Wissensformate existieren im Schweizerischen Survey-Feld? Welche Konventionen organisieren den jeweiligen Produktionsprozess?

Das erste Forschungsinteresse besteht folglich darin, unterschiedliche Survey-Welten zu identifizieren und damit einhergehend verschiedene Konventionen, welche die Survey-Produktion organisieren. Die Bearbeitung dieses Forschungsinteresses findet in Kap. 5 Die vier Survey-Welten statt. Hier werden die verschiedenen identifizierten Survey-Welten idealtypisch beschrieben.

Die Produktionsweltenperspektive und die Theorie der Rechtfertigung wurde in den vorangehenden Theoriekapiteln um die Perspektive der Quality-Chains, der Distributed Cognition und der Workplace Studies ergänzt und weiter verfeinert (Daviron und Ponte 2005; Hutchins 1996; Luff et al. 2000b). Durch die Kombination der Produktionswelten mit der Perspektive der Quality-Chains sollte eine diachrone Perspektive für die Analyse der Produktionsprozesse in den Produktionswelten ermöglicht werden. Die Quality-Chain-Perspektive bringt so einen Blick für unterschiedliche Organisationen des Produktionsprozesses ein. Unterschiedliche Konventionen, welche einen Produktionsprozess anleiten, haben folglich nicht nur eine unterschiedliche Produktqualität zur Folge, sondern bedeuten eine grundlegend andere Organisation des Produktionsprozesses (Ponte 2009). Die Theorie der Distributed Cognition, wie auch der Workplace Studies, dienen der en-detail-Analyse dieser Produktionsprozesse durch einen Fokus auf das situative und transsituative Herstellen von Survey-Qualität. Eingebracht wird hier nicht nur eine Detailperspektive auf den Produktionsprozess, sondern 
vielmehr die Frage nach einer unterschiedlichen (situativen) Mobilisierung von Entitäten (Konventionen, Formen, Objekten und Akteuren) durch verschiedene Regime des Engagements (Thévenot 2011d) und Rechtfertigungsordnungen (Boltanski und Thévenot 2007). Gefragt werden kann hier, inwiefern durch unterschiedliche Survey-Welten unterschiedliche Entitäten mobilisiert werden und welche Funktion diese für die Produktion von unterschiedlichen Wissensformate einnehmen. Folgend lässt sich das dritte Forschungsinteresse formulieren:

Wie werden produktionstechnisch und auf verschiedene Arbeitsschritte verteilt unterschiedliche Survey- und Wissensformate hergestellt? Welche (unterschiedlichen) Objekte, Formen, Regime und Konventionen ${ }^{76}$ werden für die Produktion dieser Ontologien mobilisiert und welche Funktion nehmen sie dabei ein?

Im Anschluss an das erste Forschungsinteresse stellt das zweite Forschungsinteresse eine en-detail-Perspektive auf die Herstellung von Survey-Qualität dar. Von Interesse ist hier folglich nicht alleine das Erreichen eines bestimmten Wissensformats, sondern dessen distribuierte Produktion über verschiedene Stationen hinweg. Die Bearbeitung dieses zweiten Forschungsinteresses geschieht ebenfalls in Kap. 5, indem nicht nur idealtypisch die verschiedenen Konventionen der Produktionswelten, sondern ebenfalls die spezifische (idealtypische) Organisation der statistischen Kette sowie die Epistemologien der Produktionswelten rekonstruiert werden. Die survey-pragmatische Mobilisierung von Objekten, Formen, Regimen und Konventionen stellt jedoch auch ein Thema von Kap. 6 dar. Dabei steht die Analyse der Kompromissschließung zwischen den verschiedenen Survey-Welten und den Dimensionen der Survey-Pragmatik in der Survey-Praxis im Zentrum.

Produktionswelten sind wie im vorangegangenen Kapitel dargestellt keine sich selbst vollziehenden Systeme. Mit der Unterscheidung zwischen realen und möglichen Produktionswelten verweisen Storper und Salais auf die Kombination zwischen möglichen Produktionswelten in tatsächlichen Produktionsprozessen. Die dadurch entstehenden Spannungen müssen durch eine Kompromissbildung abgefedert werden (Storper und Salais 1997, S. 41 f.). Nebst der Beschreibung der idealtypisch existierenden Survey-Produktionslogiken stellt sich deswegen die Frage nach der pragmatischen Hervorbringung und der KompromissschlieBung zwischen verschiedenen Survey-Logiken. Dadurch lässt sich das dritte Forschungsinteresse formulieren:

\footnotetext{
${ }^{76}$ Sowohl im Sinne von Dodier (Dodier 1994), als auch von Rechtfertigungsordnungen im Sinne Boltanski und Thévenots (Boltanski und Thévenot 2007).
} 
Wie werden die verschiedenen Survey-Logiken und Wissensformate in der Praxis in einen Kompromiss gesetzt? Welche praktischen Herausforderungen müssen dabei bewältigt werden, insbesondere in der interorganisationalen Koordination?

Dieses dritte Forschungsinteresse schließt an die vorherigen Forschungsinteressen an, indem danach gefragt wird, wie die verschiedenen identifizierten Survey-Welten in der Survey-Praxis zur Anwendung kommen, welche Herausforderungen sich dabei stellen und wie diese Herausforderungen durch Survey-Praktiker bewältigt werden. Adressiert werden mit diesem Forschungsinteresse folglich Kompromissschließungen auf Basis der Survey-Pragmatik, welche in Kapitel zwei als Kernkonzept der Survey-Pragmatik vorgestellt wurde. Die Bearbeitung dieses Forschungsinteresses geschieht in Kap. 6 durch die vergleichende Analyse zweier Survey-Projekte.

Diese drei ersten Forschungsinteressen dienen der Umsetzung und der empirischen Klärung einer survey-pragmatischen Perspektive auf die Survey-Forschung. Das Ziel der Forschung liegt folglich in einer pragmatischen, empirischen Prüfung der zunächst nur theoretisch beschriebenen survey-pragmatischen Perspektive auf die Survey-Forschung. Eine pragmatische Prüfung meint dabei gerade nicht ein einfaches „Testen“ der Theorie anhand der Praxis im Sinne des kritischen Rationalismus (Popper 1935). Demgemäß müsste „einfach“ geklärt werden, ob die Survey-Pragmatik der empirischen Realität entspricht oder nicht. Eine pragmatische Prüfung sieht demgegenüber in der Prüfung viel stärker einen Prozess (Strübing 2008), in welchem zwei Dinge unternommen werden. Einerseits findet eine konstante, etliche heuristische Zirkel umfassende, empiriebasierte Korrektur der ursprünglich theoretischen Konzeption statt. ${ }^{77}$ Dieser erste Schritt kann als Prozess beschrieben werden, in welchem eine Passung zwischen theoretischer Perspektive und empirischem Untersuchungsgegenstand hergestellt wird. Andererseits findet durch das empirische Material eine Detaillierung des Untersuchungsgegenstandes statt (Dewey 2008).

Nebst dieser Spezifizierung und Klärung der Survey-Pragmatik am empirischen Untersuchungsgegenstand müssen die Ergebnisse der empirischen Forschung verdichtet und mit den bestehenden Konzeptualisierungen der Survey-Produktion

\footnotetext{
${ }^{77}$ Charakteristisch für dieses Verständnis des empirischen Testens ist die methodische Vorgehensweise der Grounded Theory (Strübing 2008). Die Grounded Theory ist zwar zunächst nicht auf das Testen von vor dem Forschungsprozess existierenden Theorien ausgelegt, einem konstanten Prüfungsprozess wird hier jedoch die aus dem empirischen Material herausgearbeitete Grounded Theory unterzogen (Strauss 1998). Diese wird dadurch mit dem Fortschreiten des Forschungsprozesses immer stärker in den Daten verankert.
} 
konfrontiert werden, um einen Wissensfortschritt erreichen zu können. Dafür werden die Resultate der empirischen Forschung mit der Daten- und Prozessperspektive konfrontiert. Das leitende Forschungsinteresse in diesem Schritt lässt sich wie folgt zusammenfassen:

Was bedeutet die Produktion unterschiedlicher Wissensformate für die Qualitätsdiskussion in der Survey-Methodologie, $d$. h. sowohl für die Daten- wie auch die Prozessperspektive?

Geklärt werden soll in diesem Schritt folglich, wie unterschiedliche Wissensformate und damit einhergehend der unterschiedliche survey-weltliche Bezug auf Objekte, Formen, Regime und Konventionen ein besseres Verständnis von Qualitätsproblemen in der (praktischen) Survey-Produktion ermöglichen. Geklärt werden soll durch dieses Forschungsinteresse, welche empirisch festzustellenden Qualitätsfragen und -probleme mit der Survey-Welten-Theorie beschrieben werden können, welche durch die Daten- und Prozessperspektive nur unzureichend thematisiert werden. Thematisiert wird hier folglich der Mehrwert des Theorieansatzes für Qualitätsfragen in der Survey-Forschung. Die Bearbeitung dieses Forschungsinteresses geschieht in einem ersten Schritt in Kap. 7. Kritisiert wird hier einerseits eine Generalisierung von (erweiterten) Qualitätskriterien als Qualitätsmaßstab für verschiedene Survey-Welten. Andererseits wird die spezifische Perspektive der Survey-Welten-Theorie auf Koordinationsprobleme in Survey-Projekten dargelegt. Abschließend wird dieses Forschungsinteresse in Kap. 8 bearbeitet, indem hier anhand verschiedener Thematiken der Beitrag der Survey-Welten-Theorie zur Survey-Methodologie dargestellt wird.

Die vier beschriebenen Forschungsinteressen stellen das generelle Forschungsanliegen dieser Arbeit dar. Die Forschung ist folglich daran zu messen, inwiefern sie die hier beschriebenen Interessen konsequent verfolgt, in ein empirisches Forschungsprozedere ummünzt, beschreibt und schlussendlich infolge des vierten Forschungsinteressen auf die in Kap. 2 eröffnete Qualitätsdiskussion in der Survey-Methodologie rückspiegelt und einen Wissensgewinn ausweisen kann. Die hier beschriebenen Forschungsinteressen stellen die Grundlage für das im folgenden Kap. 4 beschriebene methodische Vorgehen dar. Aus den dargestellten Ausführungen lassen sich folgende drei Anforderungen an die methodische Umsetzung der Forschungsperspektive ableiten: Sie muss einerseits fähig sein, die epistemologischen Grundlagen verschiedener Survey-Welten zu beschreiben. Dies ist einerseits notwendig, um die ungenügende Reichweite der durch die Prozessperspektive entworfenen Qualitätskriterien aufzuzeigen und die unterschiedlichen epistemologischen Grundlagen der Survey-Produktion 
in verschiedenen Welten offenlegen zu können. Sie muss andererseits aufzeigen können, inwiefern epistemologische Grundlagen relevant sind für methodische Entscheidungen von Survey-Firmen und Auftraggeber in Survey-Projekten und diese fundieren. Sie muss folglich die situative, methodische Koordination aufarbeiten, in welchen die epistemologischen Grundlagen von Survey-Welten die situative Koordination, bzw. die methodische Praxis anleitet. Nachgezeichnet werden muss die auf Konventionen, Formen und Objekte Bezug nehmende Investitionsarbeit in das spezifische Wissensformat einer Survey-Welt, welche auf verschiedene Stationen der Produktion von statistischen Daten verteilt sein kann. Um einen Wissensfortschritt gegenüber der Prozessperspektive erzielen zu können, muss die Forschung außerdem herausarbeiten, in welchen Situationen und Qualitätswelten die durch sie beschriebenen Qualitätskriterien unzureichend und/ oder problematisch sind, bzw. eine alternative Perspektive auf Qualitätsprobleme in Survey-Projekten entwickeln. Es ist folglich notwendig, die fehlende Reichweite der Prozessperspektive am praktischen Koordinations-Engagement herauszuarbeiten.

Open Access Dieses Kapitel wird unter der Creative Commons Namensnennung 4.0 International Lizenz (http://creativecommons.org/licenses/by/4.0/deed.de) veröffentlicht, welche die Nutzung, Vervielfältigung, Bearbeitung, Verbreitung und Wiedergabe in jeglichem Medium und Format erlaubt, sofern Sie den/die ursprünglichen Autor(en) und die Quelle ordnungsgemäß nennen, einen Link zur Creative Commons Lizenz beifügen und angeben, ob Änderungen vorgenommen wurden.

Die in diesem Kapitel enthaltenen Bilder und sonstiges Drittmaterial unterliegen ebenfalls der genannten Creative Commons Lizenz, sofern sich aus der Abbildungslegende nichts anderes ergibt. Sofern das betreffende Material nicht unter der genannten Creative Commons Lizenz steht und die betreffende Handlung nicht nach gesetzlichen Vorschriften erlaubt ist, ist für die oben aufgeführten Weiterverwendungen des Materials die Einwilligung des jeweiligen Rechteinhabers einzuholen.

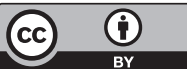

